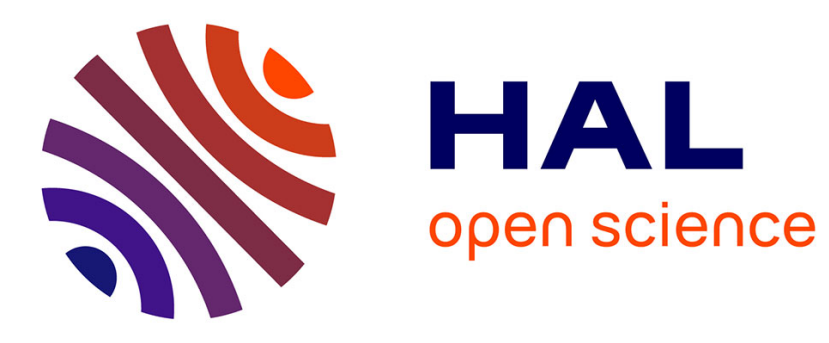

\title{
Les infortunes de la vertu
}

Xavier Timbeau, Céline Antonin, Christophe Blot, Eric Heyer, Sabine Le Bayon, Danielle Schweisguth

\section{To cite this version:}

Xavier Timbeau, Céline Antonin, Christophe Blot, Eric Heyer, Sabine Le Bayon, et al.. Les infortunes de la vertu: Perspectives économiques 2012-2013 pour l'économie européenne. 2012. hal-01069532

\section{HAL Id: hal-01069532 \\ https://hal-sciencespo.archives-ouvertes.fr/hal-01069532}

Preprint submitted on 29 Sep 2014

HAL is a multi-disciplinary open access archive for the deposit and dissemination of scientific research documents, whether they are published or not. The documents may come from teaching and research institutions in France or abroad, or from public or private research centers.
L'archive ouverte pluridisciplinaire HAL, est destinée au dépôt et à la diffusion de documents scientifiques de niveau recherche, publiés ou non, émanant des établissements d'enseignement et de recherche français ou étrangers, des laboratoires publics ou privés. 


\title{
ZONE EURO
}

\section{Les infortunes de la vertu}

\section{Perspectives économiques 2012-2013 pour l'économie européenne}

\author{
Département analyse et prévision1, \\ sous la direction de Xavier Timbeau
}

\section{Le premier pas vers la récession}

La zone euro tombera-t-elle de nouveau en récession en début d'année 2012 ? La dégradation des enquêtes de confiance après le premier trimestre 2011 (graphique 1) laissait entrevoir un nouveau repli de l'activité qui s'est confirmé au quatrième trimestre avec un recul du PIB de 0,3\%. Techniquement, la zone euro n'est pas en récession puisque la croissance du PIB était encore positive au troisième trimestre et I'amélioration des enquêtes plaide pour une moindre contraction du PIB au cours des deux premiers trimestres 2012. Cependant, au moins six pays ${ }^{2}$, représentant $33 \%$ du PIB de la zone euro, enregistraient un recul de leur activité pour le deuxième trimestre consécutif. Dans ces conditions, le répit sur le marché de l'emploi a été de courte durée. La baisse du taux de chômage observée entre le mois de mai 2010 et avril 2011 aura été bien trop faible pour absorber les effets de la crise la plus grave depuis l'aprèsguerre. En l'espace de deux trimestres, l'emploi total a reculé de 0,4\% dans la zone euro tandis que la population active augmentait de 0,2 \% portant le taux de chômage à $10,6 \%$ en décembre $2011^{3}$. Cette moyenne cache toujours une hétérogénéité croissante des situations puisque, selon les données Eurostat, le taux de chômage dépasse $23 \%$ en Espagne tandis qu'il est inférieur à 6 \% en Allemagne depuis mai 2011.

Ce recul de l'activité au quatrième trimestre s'explique principalement par une baisse de la demande intérieure (voir tableau zone euro en fin de partie), dont toutes les composantes étaient en repli. Les mesures de restriction budgétaire ont principalement pesé sur la consommation des ménages comme celle des administrations publiques qui ont baissé respectivement de $0,3 \%$ et de $0,2 \%$. Du côté de l'investissement, la baisse atteint $0,4 \%$. Elle résulte surtout de l'investissement productif, les

1. Ont contribué à cette étude: Céline Antonin, Christophe Blot, Éric Heyer, Sabine le Bayon et Danielle Schweisguth. Il intègre les informations disponibles au 27 mars 2012.

2. L'Italie, les Pays-Bas, la Belgique, le Portugal, I'Irlande et la Grèce.

3. La dégradation s'est poursuivie en janvier 2012 où le taux de chômage atteignait 10,7 \%, soit le niveau le plus élevé enregistré dans la zone euro depuis novembre 1997. 
entreprises jugeant insuffisants les débouchés au sein de la zone euro. Ce ralentissement de la demande dans la zone euro s'est répercuté sur le commerce extérieur. La faiblesse de la demande intérieure a réduit les importations de 1,5\% et donc la demande adressée à l'ensemble des pays de la zone euro, ce qui, en retour, a contraint l'évolution des exportations et a renforcé l'effet accélérateur sur l'investissement. Toutefois, la baisse des importations ayant été plus marquée que celle des exportations, la contribution du commerce extérieur à la croissance est restée positive ( 0,3 point) au quatrième trimestre 2011. Ainsi, sur l'ensemble de l'année 2011, le PIB a progressé de 1,5\% tandis que le redressement modéré de la productivité, qui a cru de $1,3 \%$, a permis une croissance timide de l'emploi de $0,2 \%$.

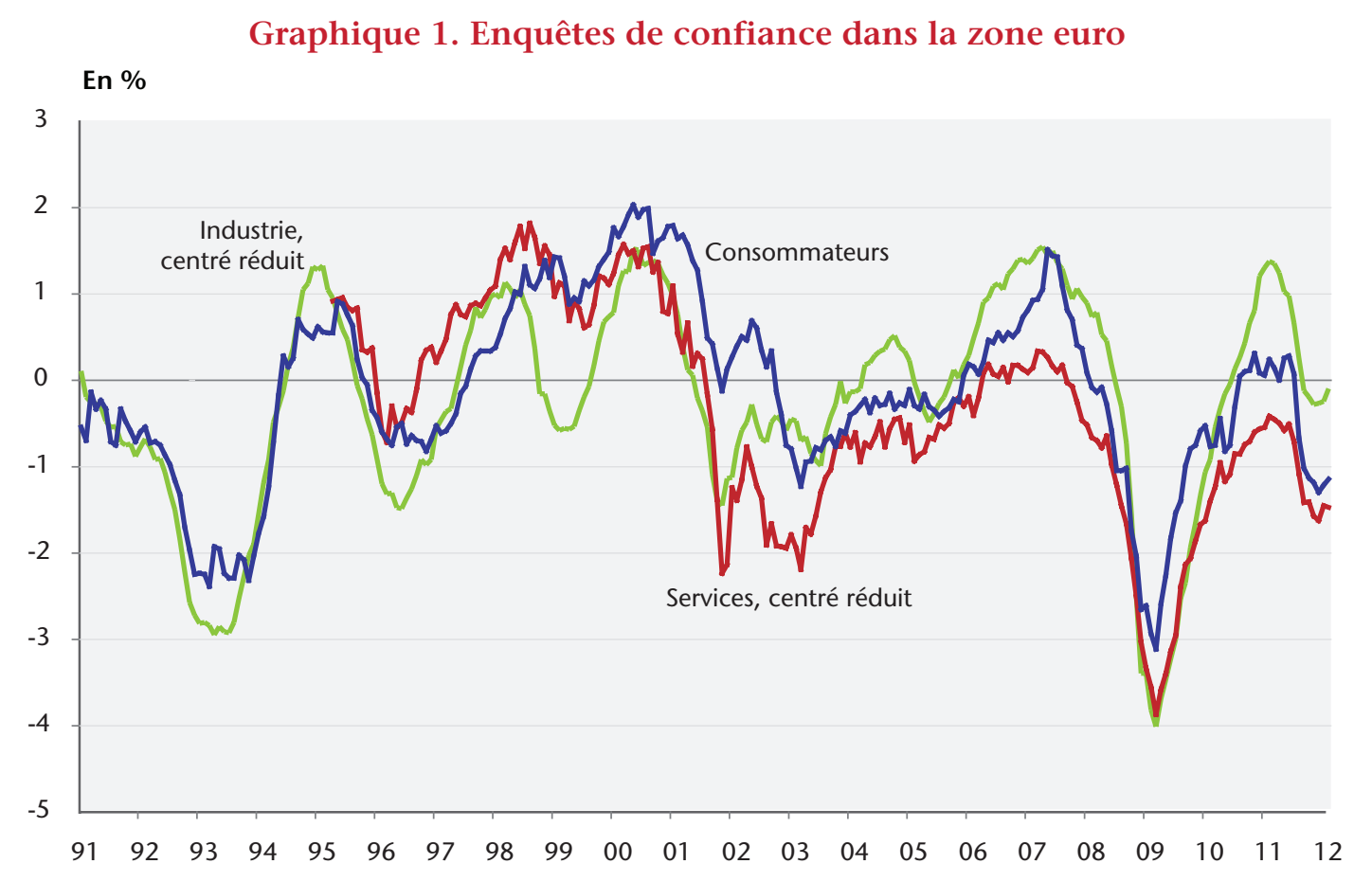

Source : Commission européenne.

\section{L'austérité confirmée...}

Pourtant, malgré ces performances insuffisantes pour refermer l'écart de production qui s'est creusé depuis la chute de l'activité intervenue en 2008, ni les gouvernements ni la Commission n'ont infléchi leur discours sur l'austérité. Comme nous l'avions souligné lors de nos prévisions ${ }^{4}$ de décembre 2011, I'interdépendance des économies européennes amplifie les effets récessifs des plans d'austérité mis en place par chacun des pays les conduisant à revoir à la baisse leurs prévisions de croissance, ce qui renforce ex-post la crise des dettes souveraines et le besoin de rigueur. L'année 2011 a été l'illustration de ce cercle vicieux dans lequel est entrée la zone euro. Les programmes d'ajustement ont pesé sur la croissance de la zone euro et en particulier sur celle des pays en crise. Mais la baisse des recettes fiscales est venue freiner la réduc-

4. Voir OFCE (2011) « La très grande Récession ", Notes de l'OFCE n 9. 
tion des déficits budgétaires en Espagne, en Irlande et au Portugal, empêchant ces pays d'atteindre leurs objectifs. Les risques d'insoutenabilité réels ou entretenus par la spéculation ont poussé les taux d'intérêt à la hausse, pénalisant la croissance et renforçant la spirale infernale. L'état de la conjoncture au quatrième trimestre semble confirmer ce constat.

Une certaine accalmie est néanmoins perceptible depuis le début de l'année 2012. Elle s'explique en partie par l'action de la BCE qui, en deux opérations exceptionnelles de refinancement, a prêté près de 1000 milliards d'euros aux banques européennes, ce qui a favorisé leur retour sur le marché des titres obligataires publics. À cet effet, il faut ajouter celui des signatures de l'accord européen pour une réforme du Pacte de stabilité et de l'accord entre la Grèce et ses créanciers privés. Néanmoins en Espagne et en Italie, deux pays n'ayant pas eu recours au FESF (Fonds européen de stabilité financière) ou à une aide européenne, les taux nominaux, bien qu'en baisse depuis le début de l'année 2012, restent proches de $5 \%$. Avec une croissance nominale qui n'a pas dépassé 2,5\% durant l'année 2011, les écarts critiques, c'est-à-dire les différences entre les taux d'intérêt et les taux de croissance potentiels nominaux, sont restés élevés (tableau 1).

Tableau 1. Évolution de l'écart critique dans les pays de la zone euro

En \% du PIB

\begin{tabular}{lcccc}
\hline & $\mathbf{2 0 0 8}$ & $\mathbf{2 0 0 9}$ & $\mathbf{2 0 1 0}$ & $\mathbf{2 0 1 1}$ \\
\hline Allemagne & 2,1 & 0,3 & 1,4 & 0,6 \\
Autriche & 0,7 & 0,9 & 0,1 & $-0,7$ \\
Belgique & 0,7 & 1,2 & 0,5 & 0,2 \\
Espagne & 0,6 & 2,3 & 2,1 & 2,6 \\
Finlande & 0,4 & 0,1 & 1,6 & $-0,9$ \\
France & 0,2 & 1,2 & 1,2 & 0,6 \\
Gèce (aide) & $-1,1$ & 1,4 & $3,1(2,5)$ & $10,0(1,1)$ \\
Irlande (aide) & 5,5 & 7,1 & 6,0 & 8,5 \\
Italie & 1,7 & 1,9 & 3,1 & 2,7 \\
Pays-Bas & 1,0 & 2,6 & 1,0 & 0,5 \\
Portugal (aide) & 1,9 & 2,5 & 2,0 & $4,1(1,1)$ \\
\hline Zone euro 11* & $\mathbf{1 , 2}$ & $\mathbf{1 , 4}$ & $\mathbf{1 , 8}$ & $\mathbf{1 , 7}$ \\
\hline
\end{tabular}

* Hors Chypre, Luxembourg, Malte, Slovaquie, Slovénie et Estonie.

Note de lecture 1 : L'écart critique est la différence entre taux d'intérêt obligataire à 10 ans et le taux de croissance potentiel nominal de l'économie (calculs OFCE).

Note de lecture 2 : Pour la Grèce, le Portugal et l'Irlande, la mention (aide) entre parenthèses indique la valeur de l'écart critique lorsque l'on considère non pas le taux d'intérêt obligataire à 10 ans sur le marché secondaire, mais le taux préférentiel auquel les pays empruntent auprès de I'UE et du FMI, fixé à 3,5 \% depuis le sommet européen de juillet 2011. Sources : Eurostat, Datastream, calculs OFCE.

La perspective d'une récession en 2012 maintiendrait cette situation, ce qui ne permettra pas d'atteindre les objectifs budgétaires. Les pays pourraient donc être contraints à de nouveaux ajustements s'ils souhaitent tenir leurs engagements et stabiliser rapidement leur dette. Comme dans notre dernière prévision 5 , nous avons simulé un scénario dans lequel les gouvernements, quelle que soit la conjoncture, respectent leurs engagements budgétaires. Si tel était le cas, cela nécessiterait de nouveaux plans de restriction budgétaire dans les mois à venir. Nous avons retenu l'hypothèse d'un 
multiplicateur budgétaire national de 0,9 correspondant à un multiplicateur total de 1,3. En effet, comme nous l'avons détaillé dans un travail récent (OFCE, 20116), la mise en place dans un contexte de basse conjoncture, de politiques de restriction budgétaire simultanées dans l'ensemble des pays européens et agissant dans une situation de faible marge de manœuvre de la politique monétaire concourt à la formation d'un multiplicateur supérieur à l'unité.

Plusieurs cas de figure ont été étudiés. De manière à isoler l'impact sur la croissance des plans d'économies nationaux et de ceux des partenaires, nous avons supposé que chaque pays respecte, seul, son engagement (tableau 2). Sous cette hypothèse, l'effort supplémentaire à fournir serait important en Italie et en Espagne : il représenterait plus de 2,1 points de PIB en 2012, et près de 1 point de PIB en 2013. Pour l'Allemagne et la France, cet effort serait faible, voire nul en 2012. II serait cependant plus conséquent en 2013 : 34,6 milliards d'euros en Allemagne et 18 milliards en France. Dans tous les cas, si tous les pays souhaitent simultanément respecter leurs engagements, ces efforts seraient fortement accrus. II faudrait compter entre 0,4/0,5 point de PIB supplémentaire en Allemagne, en France et en Italie et 0,7 point de PIB en Espagne pour I'année 2013.

Tableau 2. Impact sur le PIB du respect des engagements de réduction des déficits en 2012 et 2013

\begin{tabular}{|c|c|c|c|c|c|c|c|c|}
\hline & \multicolumn{2}{|c|}{ DEU } & \multicolumn{2}{|c|}{ FRA } & \multicolumn{2}{|c|}{ ITA } & \multicolumn{2}{|c|}{ ESP } \\
\hline & 2012 & 2013 & 2012 & 2013 & 2012 & 2013 & 2012 & 2013 \\
\hline \multicolumn{9}{|l|}{ Prévisions OFCE } \\
\hline Taux de croissance du PIB & 0,3 & 0,8 & 0,2 & 0,7 & $-1,7$ & $-0,9$ & $-1,1$ & $-0,6$ \\
\hline Solde public (en pts de PIB) & $-1,1$ & $-1,3$ & $-4,4$ & $-3,5$ & $-2,7$ & $-1,7$ & $-6,5$ & $-4,5$ \\
\hline \multicolumn{9}{|c|}{ Effort supplémentaire à fournir si chaque pays respecte seul son engagement } \\
\hline En milliards $€$ & 5,2 & 34,6 & 0,0 & 18,0 & 32,0 & 13,0 & 22,5 & 10,8 \\
\hline En points de PIB & 0,2 & 1,3 & 0,0 & 0,9 & 2,0 & 0,8 & 2,1 & 1,0 \\
\hline Taux de croissance du PIB & 0,1 & $-0,4$ & 0,0 & $-0,2$ & $-3,6$ & $-1,6$ & $-3,1$ & $-2,0$ \\
\hline Solde public (en pts de PIB) & $-1,0$ & $-0,5$ & $-4,5$ & $-3,0$ & $-1,6$ & $-0,1$ & $-5,3$ & $-3,0$ \\
\hline \multicolumn{9}{|c|}{ Effort supplémentaire à fournir si les pays de la zone euro respectent tous leurs engagements } \\
\hline En milliards $€$ & 15,7 & 45,3 & 8,0 & 28,0 & 38,5 & 19,5 & 26,8 & 18,3 \\
\hline En points de PIB & 0,6 & 1,7 & 0,4 & 1,4 & 2,4 & 1,2 & 2,5 & 1,7 \\
\hline Taux de croissance du PIB & $-0,7$ & $-1,2$ & $-0,7$ & $-1,1$ & $-4,2$ & $-2,5$ & $-3,7$ & $-3,4$ \\
\hline Solde public (en pts de PIB) & $-1,0$ & $-0,5$ & $-4,5$ & $-3,0$ & $-1,6$ & $-0,1$ & $-5,3$ & $-3,0$ \\
\hline
\end{tabular}

Sources : Prévisions et calculs OFCE, mars 2012.

5. Version actualisée (mise à jour des engagements et des déficits réalisés) du tableau 6 page 16 de « $\underline{\text { La très }}$ grande Récession ", Notes de l'OFCE, $\mathrm{n}^{\circ} 9$.

6. «La multiplication de la rigueur », Revue de l'OFCE, n 119, octobre 2011. 
Ces différents plans de rigueur nationaux, pris isolément, auraient un impact non négligeable sur la croissance des pays étudiés. En 2012, I'Allemagne et la France échapperaient à une croissance négative (respectivement $0,3 \%$ et $0,0 \%$ ). Mais une telle stratégie plongerait à nouveau I'Italie et I'Espagne dans une forte récession, avec un recul de leur PIB supérieur à 3,0 \%. En 2013 la France et I'Allemagne basculeraient à leur tour en récession: les quatre grands pays de la zone euros afficheraient des taux de croissance négatifs allant de $-0,4 \%$ pour la France et l'Allemagne, à $-2 \%$ pour l'Espagne. Bien entendu, si les grands pays de la zone euro arrivaient tous à respecter leurs engagements, alors l'effort d'économie à réaliser serait plus conséquent. Sur les deux prochaines années, il s'élèverait à près de 4 points de PIB en Italie et en Espagne, et à plus de 2 points de PIB en France et en Allemagne. Au total sur ces 4 pays étudiés, I'effort cumulé d'économies représenterait plus de 200 milliards d'euros au cours de la période 2012-2013. Le choc sur I'activité serait alors massif : il provoquerait en 2012 une récession violente pour certains pays avec une baisse du PIB de près de $4,0 \%(-4,2 \%$ en Italie et $-3,7 \%$ en Espagne). En 2013, la chute d'activité serait comprise entre -1,2\% en Allemagne et $-3,4 \%$ en Espagne.

Nous anticipons la poursuite du mouvement de détente des taux d'intérêt même s'il n'est pas exclu que de nouvelles tensions apparaissent, en lien notamment avec les chiffres de croissance ou en réaction aux difficultés des Etats membres à réduire leurs déficits budgétaires. Le taux moyen de la zone euro atteindrait 3,5\% à partir de la fin de I'année 2012, contre un pic observé à 4,5\% au deuxième trimestre 2011. Cette baisse du taux moyen résulterait d'une convergence au sein de la zone euro, comme en témoigne la baisse modérée des écarts de taux vis-à-vis de l'Allemagne observée depuis le début de l'année 2012 (graphiques 2a, b et c). Toutefois, nous ne reviendrions pas vers la situation d'absence de primes de risques qui prévalait avant l'éclatement de la crise. Surtout, cette baisse serait insuffisante pour réduire significativement les écarts critiques dans les pays fragilisés par la crise des dettes souveraines. Dans l'éventualité où les tensions se feraient plus vives que ce que nous anticipons, le besoin de rigueur budgétaire serait amplifié et le scénario de croissance devrait être revu à la baisse.

L'impulsion budgétaire7 moyenne atteindrait $-1,5$ point de PIB la première année et $-1,0$ la deuxième. En cumul sur 2012 et 2013, elle dépasserait 6 points de PIB en Espagne et 4,5 points en Italie, ce qui amputerait fortement la croissance de ces deux pays (encadré). Grâce à un déficit ramené à $1 \%$ du PIB en 2011, I'Allemagne serait peu concernée par l'ajustement budgétaire. Néanmoins, la croissance outre-Rhin serait pénalisée, via la demande adressée, par les mesures budgétaires prises dans les autres pays de la zone. Sur l'ensemble de la zone euro, il faut noter que malgré l'impact négatif de la faible activité sur le solde budgétaire conjoncturel, le déficit budgétaire serait dès 2012 ramené sous la barre de $3 \%$. Mais, par ailleurs, deux chocs négatifs supplémentaires contribueraient à entraîner la zone euro dans une nouvelle récession. D'une part, les tensions géopolitiques autour de la situation iranienne créent une forte volatilité du prix du pétrole. En dollar et en moyenne annuelle, celui-ci augmenterait de $10 \%$ en 2012 avant de se stabiliser à 120 dollars le baril en fin d'année 2013.

7. L'impulsion budgétaire est égale, aux mesures exceptionnelles près, à la variation du solde public primaire structurel. Elle donne une indication de l'orientation restrictive (en cas d'impulsion négative) ou expansionniste (en cas d'impulsion positive) de la politique budgétaire. 
Graphique 2. Écart de taux d'intérêt public à long terme vis-à-vis de l'Allemagne
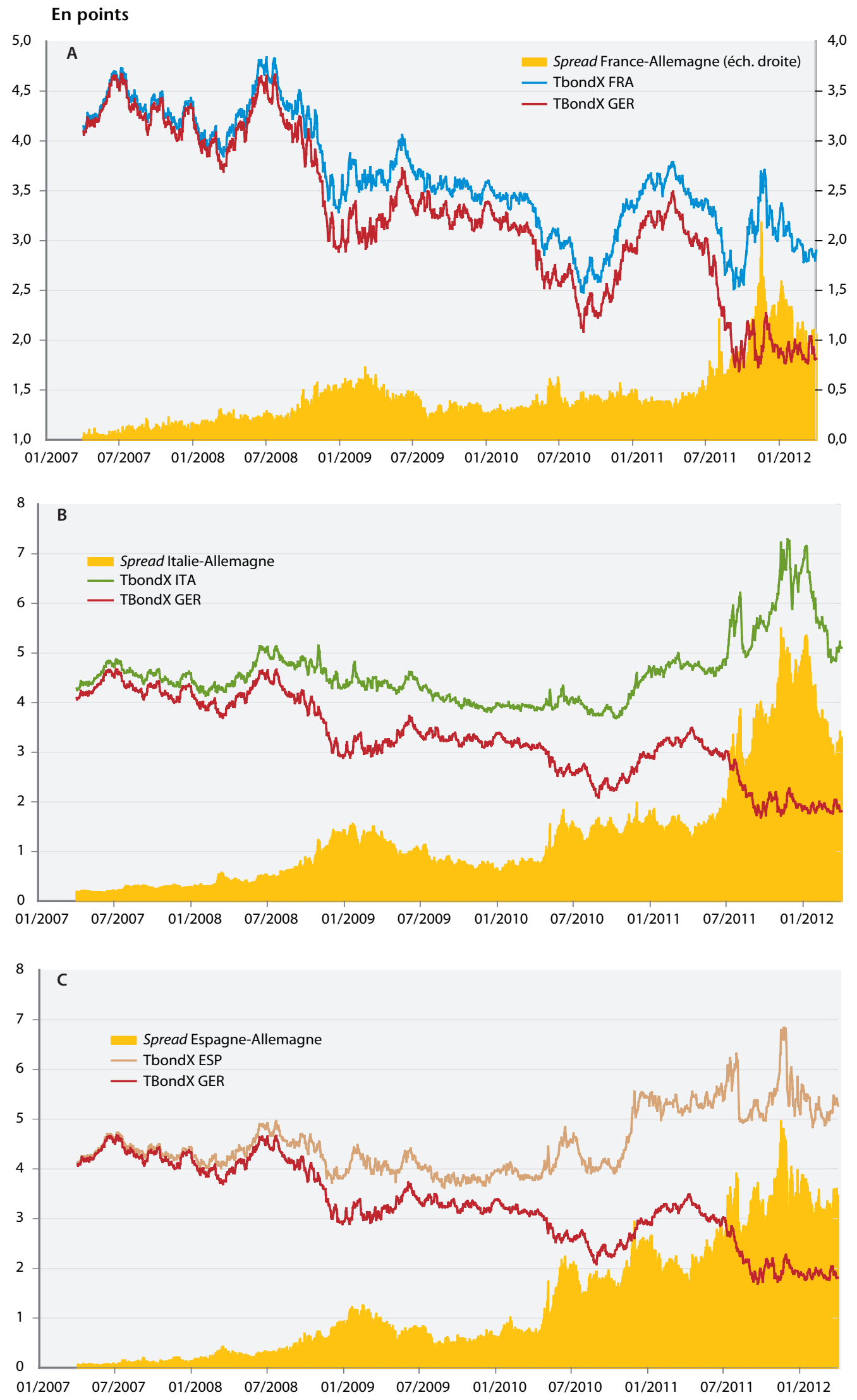

Source : Datastream. 
Exprimée en euros, cette hausse du prix du pétrole atteindrait même $20 \%$ dans la mesure où nous anticipons une dépréciation de l'euro qui s'établirait à 1,27 dollar sur l'ensemble de l'année 2012 contre 1,39 en 2011. Ensuite, la crise des dettes souveraines, la crise immobilière - en Espagne, aux Pays-Bas et en Irlande - et les faibles perspectives de croissance ont conduit les banques de la zone euro à durcir leurs conditions de crédits, comme en témoigne l'enquête publiée en janvier 2012 et réalisée sur la base de questionnaires adressés aux banques en début d'année (graphique 3). Si l'impact sur la croissance du durcissement des conditions de crédit est difficilement quantifiable, l'expérience de 2008-2009 montre que ce facteur a contribué à la récession $^{8}$. La double opération de refinancement à long terme -3 ans - menée par la BCE atténuerait ses tensions. Mais il n'en demeure pas moins que pour la France, l'effet négatif sur la croissance est estimé à 0,5\% en 2012.

Graphique 3. Conditions de crédits dans la zone euro

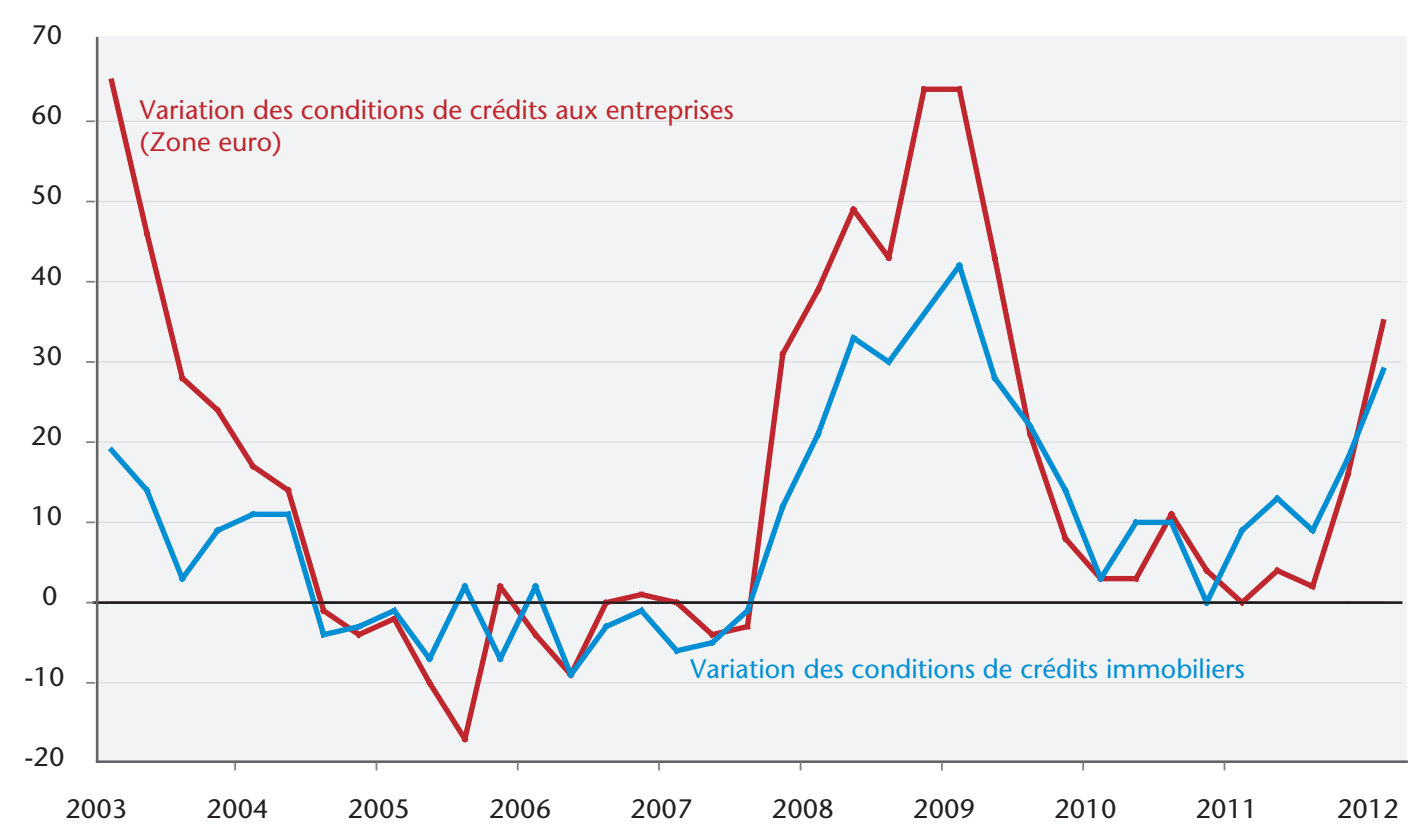

Note de lecture : les résultats sont interprétés sur la base d'un solde d'opinions entres les banques déclarant durcir leurs conditions de crédits et celles déclarant les assouplir. Une hausse de l'indicateur témoigne donc d'un durcissement des conditions de crédit.

Source : BCE. 
Le scénario de croissance pour la zone euro s'appuie sur des hypothèses relatives à l'impact des chocs budgétaires (via la demande interne et la demande adressée), pétrolier et de crédit. La croissance spontanée (avant prise en compte des chocs) résulterait de l'addition de la croissance potentielle et d'un effet de rattrapage qui dépend de l'écart de production et $d^{\prime}$ une hypothèse $a d$-hoc sur le nombre d'années nécessaires ${ }^{9}$ pour fermer cet écart. Ce rattrapage permettrait de réduire les écarts de production négatifs résultant de la crise. Les hypothèses sur la croissance potentielle et la croissance spontanée sont présentées dans le tableau E1. Les taux de croissance reportés sont annualisés et ne tiennent pas compte des acquis de croissance. Pour l'Allemagne, la croissance spontanée est évaluée à 2,3\%. Elle est moins forte qu'en France et en Espagne en raison du faible écart de production outre-Rhin. Pour les trois autres plus grands pays de la zone euro, l'effet de rattrapage est très proche (entre 1,8 et 2 points).

Tableau E1. Croissance potentielle et croissance spontanée

En \%

\begin{tabular}{|c|c|c|c|c|}
\hline & DEU & FRA & ITA & ESP \\
\hline $\begin{array}{l}\text { Croissance potentielle }(\mathrm{a})= \\
\text { Croissance de la productivité } \\
+ \text { celle de la population active }\end{array}$ & $\begin{array}{l}1,5 \\
1,1 \\
0,4\end{array}$ & $\begin{array}{r}1,45 \\
1,1 \\
0,45\end{array}$ & $\begin{array}{l}0,1 \\
0,0 \\
0,1\end{array}$ & $\begin{array}{l}1,5 \\
1,1 \\
0,4\end{array}$ \\
\hline $\begin{array}{l}\text { Output gap en } 2011 \\
\text { Nombre d'années pour fermer l'OG } \\
\text { en l'absence de chocs }\end{array}$ & $\begin{array}{l}-0,8 \\
1 \text { an }\end{array}$ & $\begin{array}{r}-6,0 \\
3 \text { ans }\end{array}$ & $\begin{array}{r}-3,6 \\
2 \text { ans }\end{array}$ & $\begin{array}{l}-7,6 \\
4 \text { ans }\end{array}$ \\
\hline Effet rattrapage annuel (b) & 0,8 & 2,0 & 1,8 & 1,9 \\
\hline $\begin{array}{l}\text { Croissance spontanée hors acquis } \\
(\mathrm{a}+\mathrm{b})\end{array}$ & 2,3 & 3,5 & 1,9 & 3,4 \\
\hline
\end{tabular}

Note : Pour l'Italie, l'estimation du taux de croissance de la productivité s'appuie sur l'évolution tendancielle observée entre 2000 et 2007. Le taux de croissance de la population active pour l'Allemagne est calé sur l'hypothèse du gouvernement allemand qui anticipe un rattrapage lié à une amélioration du taux d'emploi.

Sources: Comptes nationaux, calculs et prévision OFCE mars 2012.

Pour tenir compte des effets d'acquis, il est nécessaire de raisonner sur une base trimestrielle. Ainsi, pour la France, comme pour l'Espagne, la croissance trimestrielle spontanée estimée est proche de $0,85 \%$ (tableau E2). Elle est respectivement inférieure à 0,6 et $0,5 \%$ en Allemagne et en Italie.

Tableau E2. Impact des chocs sur la croissance trimestrielle

\begin{tabular}{|c|c|c|c|c|c|c|c|c|}
\hline & \multicolumn{2}{|c|}{ DEU } & \multicolumn{2}{|c|}{ FRA } & \multicolumn{2}{|c|}{ ITA } & \multicolumn{2}{|c|}{ ESP } \\
\hline & 2012 & 2013 & 2012 & 2013 & 2012 & 2013 & 2012 & 2013 \\
\hline Reprise spontanée & 0,58 & 0,58 & 0,86 & 0,86 & 0,48 & 0,48 & 0,85 & 0,85 \\
\hline $\begin{array}{l}\text { Impact des ajustements budgétaires } \\
\text { via la demande intérieure } \\
\text { via la demande adressée }\end{array}$ & $\begin{array}{l}-0,08 \\
-0,30\end{array}$ & $\begin{array}{l}-0,03 \\
-0,25\end{array}$ & $\begin{array}{l}-0,34 \\
-0,24\end{array}$ & $\begin{array}{l}-0,30 \\
-0,20\end{array}$ & $\begin{array}{l}-0,51 \\
-0,23\end{array}$ & $\begin{array}{l}-0,30 \\
-0,20\end{array}$ & $\begin{array}{l}-0,77 \\
-0,22\end{array}$ & $\begin{array}{l}-0,65 \\
-0,18\end{array}$ \\
\hline $\begin{array}{l}\text { Choc pétrolier } \\
\text { Autres chocs }\end{array}$ & $\begin{array}{r}-0,17 \\
0,00\end{array}$ & $\begin{array}{r}-0,04 \\
0,00\end{array}$ & $\begin{array}{l}-0,17 \\
-0,10\end{array}$ & $\begin{array}{l}-0,04 \\
-0,00\end{array}$ & $\begin{array}{l}-0,17 \\
-0,00\end{array}$ & $\begin{array}{l}-0,04 \\
-0,00\end{array}$ & $\begin{array}{l}-0,17 \\
-0,10\end{array}$ & $\begin{array}{r}-0,04 \\
0,00\end{array}$ \\
\hline Croissance trimestrielle & 0,03 & 0,25 & $-0,02$ & 0,31 & $-0,45$ & $-0,08$ & $-0,37$ & $-0,22$ \\
\hline
\end{tabular}

Sources: Comptes nationaux, calculs et prévision OFCE mars 2012.

II faut ensuite tenir compte des chocs attendus au cours des deux années 2012 et 2013. L'impact des restrictions budgétaires est décomposé entre un effet interne, qui dépend de la valeur du multiplicateur interne, et un effet externe lié aux conséquences, via la demande adressée, des plans d'ajustement mis en œuvre dans le reste du monde. L'essentiel de cet effet résulte des impulsions négatives des pays de l'Union européenne.

9. Au cours des crises précédentes, il ressort que la vitesse de fermeture des écarts de production est généralement comprise entre 1 et 5 années en fonction du niveau de cet écart. 
C'est en Allemagne que l'effet interne est le plus faible alors qu'il atteint $-0,8$ point en 2012 en Espagne. En revanche l'Allemagne serait plus fortement touchée par l'effet externe qui atteindrait 0,30 point en 2012 contre 0,2 dans les trois autres pays. Pour ces quatre pays de la zone euro, nous supposons que l'effet du choc pétrolier serait identique en 2012 et 2013. Enfin, l'effet des autres chocs et notamment du durcissement des conditions de crédit serait de l'ordre de 0,1 point de croissance trimestrielle en France et en Espagne en 2012.

Sur la base de ces hypothèses, la croissance trimestrielle moyenne en 2012 et 2013 serait négative en Espagne et en Italie. Elle serait très proche de zéro en France et en Allemagne en 2012, et serait voisine de 0,3\% en 2013. C'est donc grâce à un effet d'acquis favorable que la France échapperait à la récession sur l'ensemble de l'année 2012 puisque la croissance annuelle y atteindrait $0,2 \%$. Inversement, l'effet d'acquis contribuerait à amplifier la récession de I'Italie et de I'Espagne où le recul du PIB atteindrait respectivement 1,7 et $1,1 \%$.

\section{... la récession aussi}

Le PIB de la zone euro reculerait de 0,2\% au premier trimestre 2012 et encore de $0,1 \%$ au trimestre suivant. Le poids conjugué des trois chocs (budgétaires, pétrolier et de crédit) pèserait principalement sur la demande intérieure. La consommation des ménages reculerait en 2012 et resterait anémique en 2013. L'absence de débouchés ainsi que le faible niveau du taux d'utilisation des capacités de production, qui est repassé sous sa moyenne de long terme au deuxième semestre 2011, freinerait l'investissement productif dont le recul atteindrait 1,5\% en 2012 (graphique 4). De plus, les effets liés au durcissement des conditions de crédit se répercuteraient notamment sur I'investissement-logement, qui reculerait de 0,9\% en 2012 et de 0,3\% l'année suivante.

\section{Graphique 4. Effet accélérateur sur l'investissement}

$$
\text { En g.a (en \%) }
$$

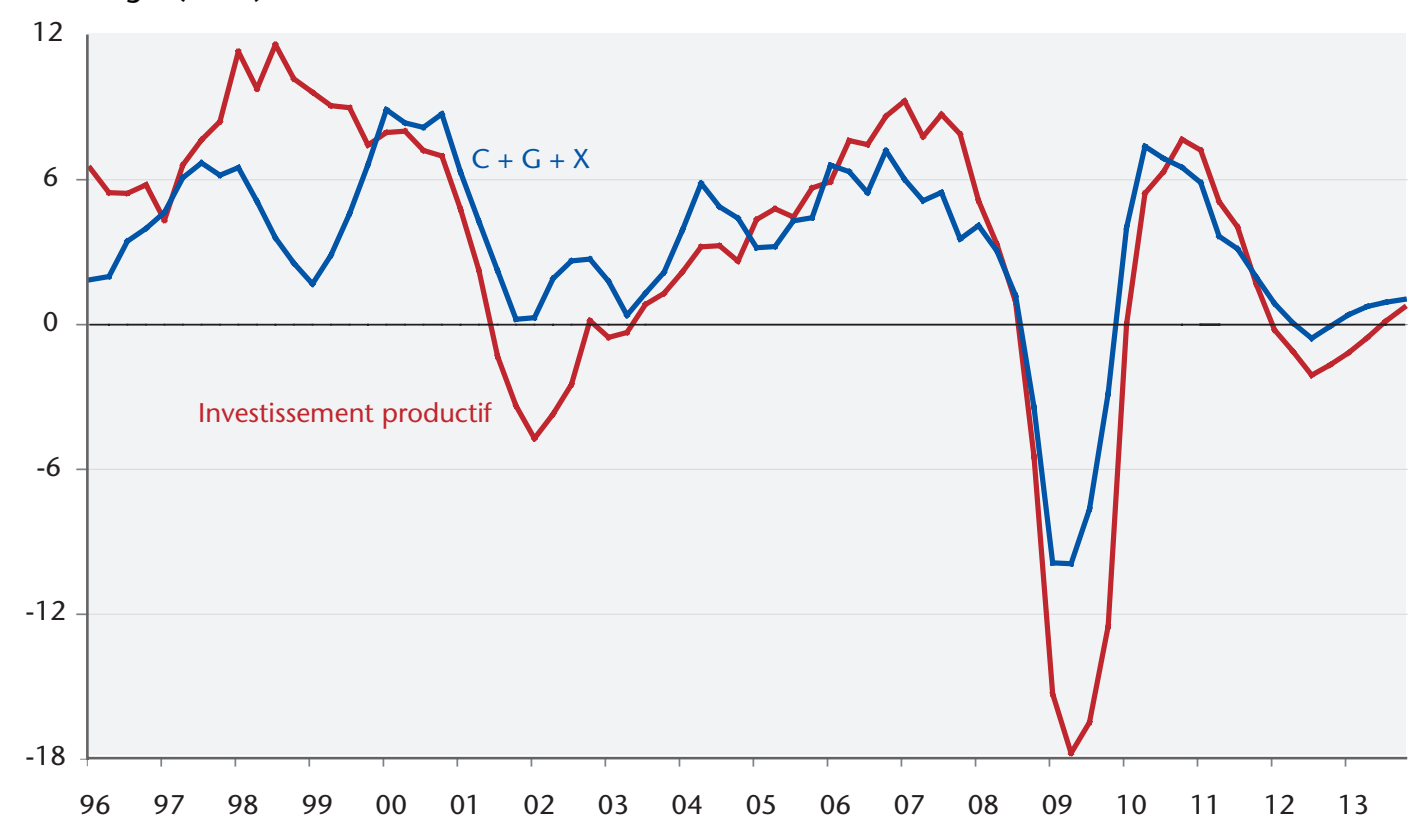

Note: l'effet accélérateur résulte de la relation entre la croissance de l'investissement et celle de la demande mesurée ici par la somme des consommations finales (publiques $G$ et privées $C$ ) et des exportations $\mathrm{X}$.

Source : Eurostat, prévision OFCE mars 2012. 
L'impact global de ces chocs serait ensuite amplifié via la demande adressée si bien que malgré la contribution positive du commerce extérieur de l'ensemble de la zone euro, le taux de croissance des exportations ne dépasserait pas $1 \%$ en 2012 et s'élèverait à 1,5\% en 2013. Sur l'ensemble de l'année 2012, le PIB de la zone euro diminuerait de 0,4\%. L'Espagne, I'Italie, les Pays-Bas, I'Irlande, le Portugal et la Grèce seraient en récession (tableau 3). L'ampleur du recul de l'activité serait moins forte que celle observée en 2009, mais il reste que, fin 2013, le niveau d'activité serait toujours inférieur à celui qui prévalait au 1 er trimestre 2008, éloignant à nouveau la perspective d'une amélioration durable de l'emploi. Le taux de chômage continuerait d'augmenter et atteindrait 11,1\% en fin d'année 2013, soit le niveau le plus élevé depuis la création de l'euro. Du côté des prix, l'inflation se maintiendrait toujours au-dessus de la cible visée par la BCE en 2012. Malgré la hausse du prix du pétrole, le taux de croissance des prix serait moins élevé qu'en 2011 et la BCE ne devrait donc pas durcir sa politique monétaire. L'impact négatif sur les salaires du niveau élevé du chômage conduirait à une baisse de l'inflation sous-jacente. En moyenne annuelle, le taux d'inflation s'établirait à 2,3\% en 2012 et 1,9\% en 2013.

La zone euro serait toujours coupée en deux ${ }^{10}$ puisque la divergence des trajectoires de reprise observée en 2010 et en début d'année 2011 s'amplifierait (graphique 5). En effet, avant l'éclatement de la crise au deuxième trimestre 2008, le PIB par tête de I'Italie et de I'Espagne était inférieur à celui de l'Allemagne de respectivement 12 et $13 \%$. Fin 2013, ces écarts s'élèveraient à 23,4\% pour I'Italie et $22,8 \%$ pour I'Espagne. L'écart de PIB par tête entre la France et I'Allemagne passerait de $7 \%$ à $11,6 \%$ sur la même période.

Tableau 3. Croissance des principaux pays de la zone euro

\begin{tabular}{lccc} 
En \% & 2011 & 2012 & 2013 \\
\hline Allemagne & 3,1 & 0,3 & 0,8 \\
France & 1,7 & 0,2 & 0,7 \\
Italie & 0,5 & $-1,7$ & $-0,9$ \\
Espagne & 0,7 & $-1,1$ & $-0,6$ \\
Pays-Bas & 1,3 & $-1,1$ & 0,5 \\
Belgique & 1,9 & 0,1 & 0,9 \\
Irlande & 0,7 & $-0,3$ & 0,9 \\
Portugal & $-1,5$ & $-2,9$ & 0,2 \\
Grèce & $-6,2$ & $-4,6$ & 0,3 \\
Finlande & 2,7 & 0,7 & 1,1 \\
Autriche & 3,1 & 0,4 & 0,8 \\
Zone euro & 1,5 & $-0,4$ & 0,3 \\
\hline
\end{tabular}

Sources : Eurostat, calculs et prévision OFCE mars 2012.

10. Voir OFCE (2011) : «Zone euro : coupée en deux », Revue de I'OFCE, n 117, p. 43-89. 
Dans ce contexte essentiellement marqué par la mauvaise synchronisation des politiques budgétaires, le soutien de la politique monétaire serait essentiel mais limité. Essentiel car l'action de la BCE en tant que prêteur en dernier ressort réduit les risques d'illiquidité et donc de faillite d'une banque européenne ce qui ne manquerait pas, autrement, de relancer le risque systémique. Mais le rôle de la BCE est limité car elle dispose de peu marge de manœuvre pour baisser les taux d'intérêt à court terme et elle ne s'engage pas dans une politique massive d'achats de titres sur les marchés obligataires publics qui serait le seul moyen d'exercer une pression rapide et durable sur les taux d'intérêt à long terme. Dans ces conditions, la capacité de rebond des économies européennes repose sur l'ajustement déflationniste mis en œuvre par le biais des restrictions budgétaires et par la réduction, plus ou moins rapide, des écarts de production. Les effets de cette stratégie tardent cependant à se faire sentir. Les ajustements de coûts salariaux et de compétitivité sont en effet lents à opérer dans une zone monétaire fortement intégrée alors que les effets dépressifs sur la demande sont importants et immédiats.

\section{Graphique 5. PIB par tête}

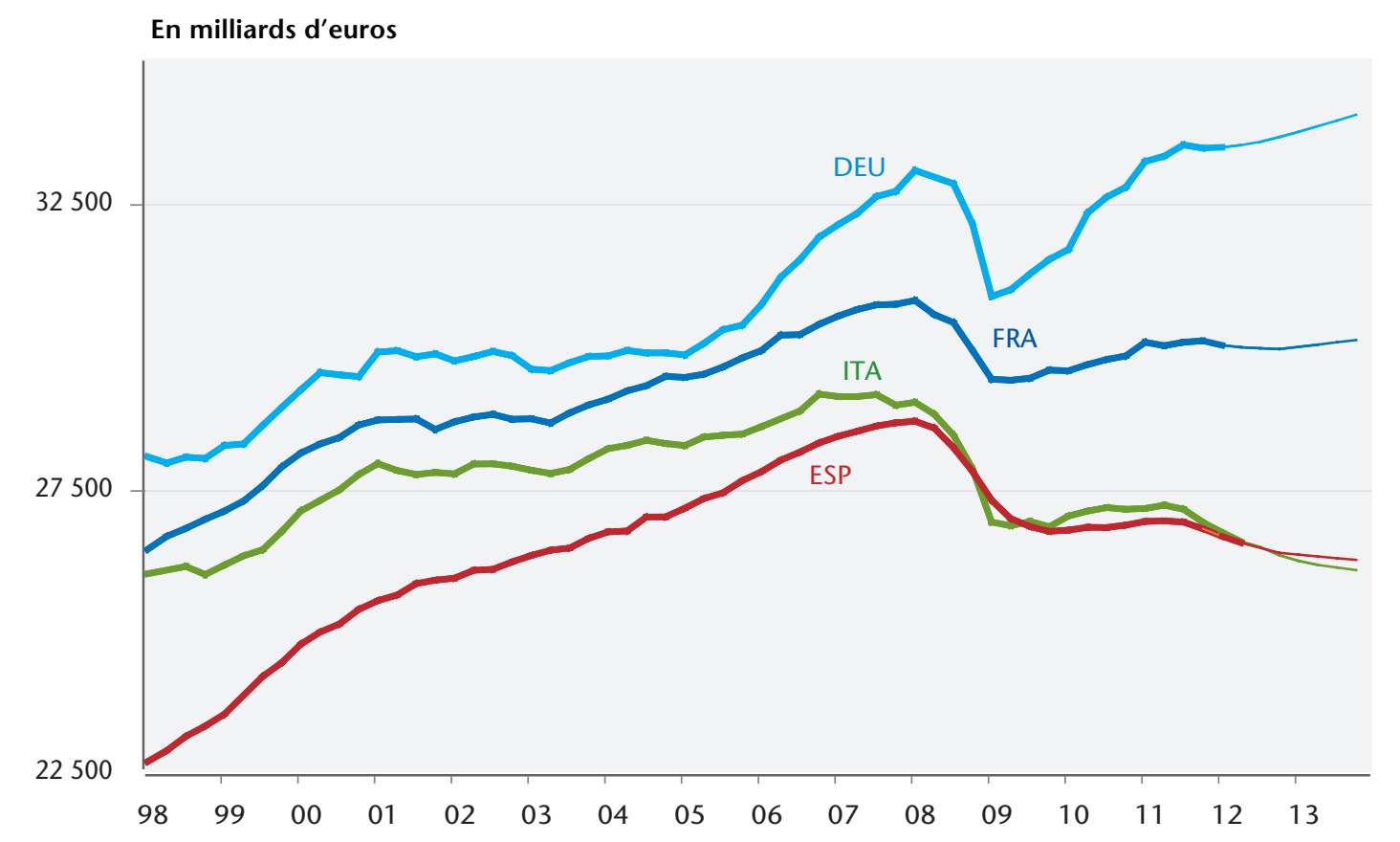

Sources : Comptes nationaux, calculs et prévisions OFCE, mars 2012.

\section{Finances publiques : surenchère dans l'austérité}

Alors qu'en 2010, certains pays de la zone euro - I'Allemagne, I'Autriche ou I'Irlande - n'avaient pas encore amorcé de politique de réduction des déficits, I'année 2011 a été celle d'une cure d'austérité budgétaire généralisée. Ainsi, le déficit public de la zone euro s'est réduit de près de 2 points, passant de 5,5 à 3,6\% du PIB (tableau 4). Pour 2012 et 2013, les gouvernements ont annoncé une intensification de la rigueur budgétaire, avec des objectifs de déficit particulièrement ambitieux, difficiles à atteindre dans un contexte de croissance molle. 
La nécessité de revenir, à marche forcée, dans les limites du Pacte de stabilité et de croissance a plusieurs origines :

1) L'inquiétude suscitée par la hausse des taux directeurs sur les titres obligataires souverains en fin d'année 2011 et le creusement de l'écart avec le taux allemand, pour des pays jusqu'alors épargnés par la crise (Italie, Espagne, et même France dans une moindre mesure). Le creusement de l'écart critique et le risque d'emballement de la dette jette le doute sur la soutenabilité des finances publiques de certains pays de la zone.

2) Les craintes d'une hausse du coût du financement de la dette ont également été alimentées par la dégradation de la note de plusieurs pays de la zone euro par l'agence Standard and Poor's. Cette dernière a en effet décidé, en janvier 2012, de retirer le triple A de la France et de l'Autriche, et d'abaisser les notes de l'Espagne, de I'Italie, du Portugal et de Chypre, plaçant ces deux derniers pays en catégorie spéculative.

3) La crainte de contagion du cas grec est toujours présente, malgré le vote du deuxième plan d'aide qui représente un total de 130 milliards d'euros, garantis par les pays membres.

Tableau 4. Évolution du solde public dans les pays de la zone euro

En \% du PIB

\begin{tabular}{lccccccc} 
& 2007 & 2008 & 2009 & 2010 & 2011 & 2012 & 2013 \\
\hline Allemagne & 0,2 & $-0,1$ & $-3,2$ & $-4,3$ & $-1,0$ & $-1,1$ & $-1,3$ \\
Autriche & $-0,9$ & $-0,9$ & $-4,1$ & $-4,4$ & $-3,4$ & $-3,0$ & $-3,0$ \\
Belgique & $-0,3$ & $-1,3$ & $-5,8$ & $-4,1$ & $-4,0$ & $-3,4$ & $-3,0$ \\
Espagne & 1,9 & $-4,5$ & $-11,2$ & $-9,3$ & $-8,5$ & $-6,5$ & $-4,5$ \\
Finlande & 5,3 & 4,3 & $-2,5$ & $-2,5$ & $-1,2$ & $-0,9$ & $-0,6$ \\
France & $-2,7$ & $-3,3$ & $-7,5$ & $-7,1$ & $-5,4$ & $-4,4$ & $-3,5$ \\
Grèce & $-6,5$ & $-9,8$ & $-15,8$ & $-10,6$ & $-9,3$ & $-7,3$ & $-4,6$ \\
Irlande & 0,1 & $-7,3$ & $-14,4$ & $-32,0$ & $-10,1$ & $-8,7$ & $-7,5$ \\
Italie & $-1,6$ & $-2,7$ & $-5,4$ & $-4,6$ & $-3,8$ & $-2,8$ & $-1,7$ \\
Pays-Bas & 0,2 & 0,5 & $-5,6$ & $-5,1$ & $-5,0$ & $-4,5$ & $-4,0$ \\
Portugal & $-3,1$ & $-3,6$ & $-10,1$ & $-9,8$ & $-4,0$ & $-4,5$ & $-3,5$ \\
Zone euro $11^{*}$ & $-0,6$ & $-1,8$ & $-5,5$ & $-5,5$ & $-3,6$ & $-2,9$ & $-2,3$ \\
\hline
\end{tabular}

*Hors Chypre, Luxembourg, Malte, Slovaquie, Slovénie et Estonie. Sources : Eurostat pour 2007 à 2011, prévisions OFCE mars 2012.

Tous les pays ont inscrit une cure d'austérité budgétaire pour 2012-2013, avec des impulsions fortement négatives (tableau 5). Une austérité budgétaire trop forte et prolongée pourrait donc avoir l'effet pervers de réduire la croissance à court et à moyen terme, et donc de rendre plus difficile la sortie de crise par le haut (via le retour de la croissance). En outre, l'analyse des écarts fiscaux (tax gap, tableau 6) montre que I'Italie a déjà réalisé les ajustements structurels nécessaires pour stabiliser la dette brute dès 2011, suivie de la France et l'Allemagne en 2012, et de I'Espagne en 2013. En ce qui concerne les petits pays, la Grèce a déjà réalisé ses ajustements en 2011, suivie du Portugal en 2012. Seule l'Irlande ne devrait pas réussir à stabiliser son ratio d'endettement à l'horizon de 2013. 
Tableau 5. Impulsions budgétaires des pays de la zone euro

En points de PIB

\begin{tabular}{lccccc} 
& 2009 & $\mathbf{2 0 1 0}$ & $\mathbf{2 0 1 1}$ & $\mathbf{2 0 1 2}$ & $\mathbf{2 0 1 3}$ \\
\hline Allemagne & 0,7 & 1,2 & $-0,9$ & $-0,3$ & $-0,1$ \\
Autriche & 0,4 & 0,6 & $-0,5$ & $-1,2$ & $-0,5$ \\
Belgique & 1,8 & $-0,3$ & $-0,1$ & $-1,4$ & $-0,8$ \\
Espagne & 4,1 & $-1,9$ & $-1,2$ & $-3,4$ & $-2,9$ \\
Finlande & 0,5 & 1,5 & $-1,1$ & $-1,1$ & $-1,3$ \\
France & 2,5 & $-0,7$ & $-1,9$ & $-1,4$ & $-1,5$ \\
Grèce & 1,8 & $-7,9$ & $-5,0$ & $-4,3$ & $-3,2$ \\
Irlande & 3,7 & $-4,1$ & $-2,5$ & $-3,0$ & $-2,6$ \\
Italie & 0,8 & $-0,4$ & $-1,2$ & $-2,9$ & $-1,7$ \\
Pays-Bas & 3,8 & $-1,5$ & $-0,2$ & $-1,9$ & $-1,0$ \\
Portugal & 4,9 & $-0,6$ & $-3,8$ & $-3,0$ & $-3,0$ \\
\hline Zone euro 11 & 1,6 & $-0,3$ & $-1,1$ & $-1,5$ & $-1,0$ \\
\hline
\end{tabular}

*Hors Chypre, Luxembourg, Malte, Slovaquie, Slovénie et Estonie,

Note de lecture : L'impulsion budgétaire est mesurée par l'opposé de la variation du solde structurel primaire, c'est-à-dire hors charges d'intérêt et recettes exceptionnelles : elle est une approximation de la politique budgétaire discrétionnaire. Sources : Calculs et prévisions OFCE, mars 2012.

\section{Tableau 6. Écarts fiscaux (tax gap)}

En \% du PIB

\begin{tabular}{lrrrrrr} 
& 2008 & 2009 & 2010 & 2011 & $\mathbf{2 0 1 2}$ & 2013 \\
Allemagne & $-0,7$ & $-0,8$ & 1,4 & 0,4 & $-2,1$ & $-1,6$ \\
France & 0,6 & 3,9 & 3,2 & 1,4 & $-0,9$ & $-1,6$ \\
Italie & 0,2 & 0,2 & 1,7 & $-0,1$ & $-2,5$ & $-3,5$ \\
Espagne & 2,3 & 7,9 & 5,1 & 3,1 & 0,7 & $-1,3$ \\
Irlande & 7,6 & 12,7 & 29,1 & 6,0 & 2,8 & 0,9 \\
Portugal & 1,8 & 7,5 & 6,5 & 2,6 & $-1,9$ & $-3,4$ \\
Grèce & 3,8 & 9,2 & 2,7 & $-1,8$ & $-5,8$ & $-7,8$ \\
\hline
\end{tabular}

Note de lecture : L'écart fiscal (tax gap) représente l'augmentation de taxes (ou la baisse de dépenses) nécessaire pour stabiliser la dette publique brute à moyen terme. Lorsque l'écart fiscal devient négatif (en gras), cela indique que le pays a procédé à l'ajustement nécessaire pour stabiliser sa dette.

Sources : OCDE, Eurostat, prévision OFCE, mars 2012.

Note méthodologique: Dans ce scénario, nous utilisons le solde public, le taux de croissance du PIB et le taux d'intérêt apparent sur la dette publique issus des prévisions OFCE. Nous faisons I'hypothèse d'une fermeture progressive de l'écart de production à partir de 2013 selon la formule suivante :

$$
O G_{t}=O G_{t-1}+1+\frac{1}{10}\left(O G_{t=c r e u x O G}\right)+\text { impulsion budgétaire }
$$

Ainsi, à partir de 2013, nous prévoyons une fermeture de l'écart de production d'un point de PIB potentiel par an, plus un dixième de l'écart de production (mesuré l'année où l'écart de production est le plus dégradé), plus le montant de l'impulsion budgétaire.

Note de lecture: L'écart fiscal (tax gap) représente l'augmentation de taxes (ou la baisse de dépenses) nécessaire pour stabiliser la dette publique brute à moyen terme. Lorsque l'écart fiscal devient négatif (en gras), cela indique que le pays a procédé à l'ajustement nécessaire pour stabiliser sa dette. 


\section{À l'impossible, nul ne devrait être tenu}

Le ralentissement de l'activité, plus fort que celui anticipé par les différents gouvernements, creuse le solde public conjoncturel et ne permet pas à tous les pays de respecter leur engagement. Cela sera notamment le cas, d'après nos prévisions, pour les gouvernements italien et espagnol qui pourraient être confrontés à un écart entre le solde public réalisé et leur engagement compris entre 1,1 et 1,5 points de PIB au cours des deux prochaines années. Dans le cas de la France et de l'Allemagne, les engagements seraient tenus en 2012 mais l'écart avoisinerait 0,5-0,7 point en 2013 (tableau 7).

Au sein des grands pays de la zone euro, la divergence est particulièrement marquée entre une Allemagne dont l'ajustement budgétaire a été réalisé avec succès, et les pays du Sud (Italie et Espagne) qui devraient plonger en récession sous l'effet d'impulsions budgétaires très négatives. L'Allemagne a connu une amélioration franche de son déficit public en 2011, qui a atteint $1 \%$ du PIB, après $4,3 \%$ en 2010 . La forte croissance, l'absence de mesures exceptionnelles (contre 1,3 point de PIB en 2010) et la restriction budgétaire ( 0,9 point) ont permis à l'Allemagne de dépasser ses objectifs de déficit. Ceci lui permet de mener une politique budgétaire beaucoup moins restrictive que la plupart des pays de la zone euro (-0,3 point prévu en 2012, -0,1 point en 2013). Le gouvernement a ainsi annoncé récemment des mesures pour soutenir le revenu des ménages et l'investissement public, limitant la consolidation programmée en juillet 2010. Face à la faiblesse de la croissance, la restriction n'empêcherait pas le déficit de se creuser, à $1,1 \%$ du PIB en 2012 et 1,3\% en 2013 (contre 0,5\% prévu par le gouvernement pour 2013). La dette publique se stabiliserait autour de $80 \%$ du PIB.

Tableau 7. Solde public en 2012 et 2013 prévu par...

En points de PIB

\begin{tabular}{lcccccccc} 
& \multicolumn{2}{c}{ DEU } & \multicolumn{2}{c}{ FRA } & \multicolumn{2}{c}{ ITA } & \multicolumn{2}{c}{ ESP } \\
& 2012 & 2013 & 2012 & 2013 & $\mathbf{2 0 1 2}$ & $\mathbf{2 0 1 3}$ & $\mathbf{2 0 1 2}$ & $\mathbf{2 0 1 3}$ \\
\hline$\ldots$.. I'OFCE & $-1,1$ & $-1,3$ & $-4,4$ & $-3,5$ & $-2,7$ & $-1,6$ & $-6,5$ & $-4,5$ \\
... les gouvernements & $-1,0$ & $-0,5$ & $-4,5$ & $-3,0$ & $-1,6$ & $-0,1$ & $-5,3$ & $-3,0$ \\
\hline Écart & $\mathbf{0 , 1}$ & $\mathbf{0 , 7}$ & $\mathbf{0 , 1}$ & $\mathbf{0 , 5}$ & $\mathbf{1 , 1}$ & $\mathbf{1 , 5}$ & $\mathbf{1 , 2}$ & $\mathbf{1 , 5}$ \\
\hline
\end{tabular}

Sources : Sources nationales, prévisions OFCE.

En 2010, la France a amorcé le long chemin de rétablissement des comptes public, le déficit passant de 7,5\% du PIB en 2009 à 7,1 \% en 2010. En 2011, le solde public s'établirait à 5,2\% du PIB. La baisse du déficit public s'est accélérée en 2011 sous l'effet de la hausse de la pression fiscale, liée notamment aux mesures de réduction des niches fiscales et sociales, du rebond spontané particulièrement marqué de certaines recettes, en particulier de l'impôt sur les sociétés, et de la fin de l'ensemble des mesures du plan de relance. En 2012, sur la base du cadrage budgétaire prévu par le gouvernement, les taux de prélèvements obligatoires augmenteraient de 1,1 point de PIB (poursuite de la réduction des niches fiscales et mesures fiscales du plan d'austérité du plan du 7 novembre) permettant une réduction du déficit public de 0,8 point de PIB malgré la faible croissance (0,2\%). En 2013, selon le gouvernement, le taux de prélèvements obligatoires continuerait à augmenter de 0,5 point de PIB et les dépenses publiques connaîtraient un coup d'arrêt historique malgré la hausse des charges d'inté- 
rêts (croissance négative en volume de la dépense publique primaire prévue pour 2013). La réduction du déficit public structurel serait de 1,5 point de PIB et celle du déficit public de «seulement »0,9 point de PIB en raison de la faible croissance $(0,7 \%)$. II atteindrait 3,5\% du PIB en 2013. Sur la période 2011-2013, la hausse des taux de prélèvements obligatoires serait de près de 3 points de PIB $(44,9 \%$ du PIB, soit un retour au niveau de 1999) et la réduction du déficit structurel de 4,5 points de PIB. En revanche, cette politique budgétaire d'intense rigueur aurait pour effet d'effacer la reprise spontanée de la croissance sur la période 2011-2013, creusant ainsi l'output gap et les déficits publics conjoncturels (-1,2 point de PIB).

Après les trois plans de rigueur votés en 2011, I'Italie devrait connaître une année 2012 marquée par une violente austérité budgétaire (avec 49 milliards d'euros d'économies, voir Italie : Le défi de Mario Monti). Outre le gel des salaires des fonctionnaires jusqu'en 2013, la baisse des effectifs dans le secteur public, la baisse des budgets dans les ministères et des dépenses dans le secteur de la santé décidés en juillet et août 2011, le plan de décembre 2011 ajoute la réforme du régime de retraite. La rigueur passera essentiellement par la hausse des recettes, avec l'augmentation des droits d'accises, la hausse de la fiscalité directe, la lutte contre l'évasion fiscale, la révision et la réévaluation du cadastre, l'augmentation de deux points de la TVA à taux normal et à taux réduit fin 2012 et une nouvelle taxation sur les produits financiers, sur les comptes courants bancaires et sur les comptes de dépôt. Les trois plans d'austérité, qui représentent un total de 49 milliards d'euros en 2012 et 76 milliards en 2013, se traduiront par des impulsions budgétaires très négatives (respectivement $-2,9$ et $-1,7$ point), plongeant ainsi le pays dans la récession. Etant donné cette récession, et l'augmentation du taux d'intérêt sur la dette publique italienne (6\% en 2012), l'objectif du gouvernement d'atteindre l'équilibre budgétaire en 2013 ne devrait pas être réalisé, et le déficit se réduirait plus lentement pour atteindre 1,7 \% du PIB en 2013.

Après d'intenses négociations avec la Commission européenne, le premier ministre espagnol Mariano Rajoy a obtenu une révision de l'objectif de déficit public espagnol pour l'année 2012 à 5,3\% du PIB, au lieu de 4,4\% initialement prévus. Cet objectif est cependant inférieur à celui qu'avait annoncé le premier ministre espagnol lorsqu'ont été publiés les chiffres de déficit pour l'année 2011, qui faisaient état d'une dégradation beaucoup plus importante qu'anticipé du déficit public $(8,5 \%$ du PIB alors que l'objectif pour l'année 2011 était de $6 \%$ ). Mariano Rajoy avait alors expliqué qu'il devenait très difficile pour l'Espagne de satisfaire l'objectif de 4,4\% en 2012, et s'était fixé un objectif de 5,8\%. La Commission a insisté pour que l'objectif de $3 \%$ en 2013 soit maintenu et a accepté de réduire à 5,3\% l'objectif de 2012. L'effort budgétaire à accomplir pour diminuer de plus de trois points le déficit public en 2012 (de 8,5 à $5,3 \%)$ s'élève à près de 35 milliards d'euros. Le 30 décembre, le gouvernement espagnol a annoncé un plan de rigueur de l'ordre de 15 milliards, qui inclue le gel des salaires des fonctionnaires et une hausse de leur temps de travail, le non-remplacement des départs à la retraite dans les administrations et des hausses d'impôt sur le revenu et le patrimoine (de $0,75 \%$ à $7 \%$ selon la tranche). Puis le 3 avril, le gouvernement de Marianno Rajoy a surenchéri avec un plan d'austérité de 27,3 milliards d'euros. Les mesures supplémentaires annoncées incluent une hausse de l'imposition des sociétés qui devrait rapporter 5,3 milliards d'euros, une amnistie fiscale avant un durcissement des mesures anti-fraude de façon à inciter les particuliers à déclarer la totalité de leurs revenus (gains espérés: 2,5 milliards d'euros), et une baisse des dépenses dans les ministères de $17 \%$ (13 milliards d'euros). Le reste de l'ajustement proviendra de 
l'effort budgétaire fourni par les communautés autonomes. L'impact négatif du plan de rigueur sur le PIB espagnol entraînera une récession en 2012 qui se traduira par un manque à gagner en termes de recettes fiscales. Selon nos prévisions, l'Espagne ne parviendrait pas à respecter ses engagements budgétaires en 2012 et le déficit s'élèverait à $6,5 \%$ du PIB. La rigueur budgétaire sera toujours de mise en 2013. Le gouvernement pourrait alors annoncer un nouveau plan de rigueur de l'ordre de 30 milliards d'euros, incluant probablement une nouvelle hausse de TVA, après celle de 2010. Selon nos calculs, l'Espagne serait de nouveau en récession en 2013 et le déficit se situerait autour de $4,5 \%$ du PIB, soit une consolidation par rapport au niveau de $2011(-8,5 \%)$.

Dans les pays périphériques, la récession devrait se prolonger au Portugal et en Grèce, rendant la réduction des déficits d'autant plus douloureuse.

\section{Grèce : prisonnière de la récession}

Outre la nécessité de recapitaliser les banques en injectant 106 milliards d'euros d'ici le 30 juin 2012 pour permettre aux fonds propres «durs » d'atteindre $9 \%$ (mise en conformité avec Bâle III), le sommet européen du 26 octobre 2011 a marqué une avancée majeure sur la résolution de la crise grecque. II a en effet été décidé de restructurer la dette grecque, pour la ramener de plus de $160 \%$ du PIB à $120 \%$ en 2020, un niveau jugé supportable par les autorités européennes. Pour permettre cette restructuration, un plan en deux volets a été mis en place, avec une participation conjointe des créanciers privés de la Grèce, via le Private Sector Involvement (PSI, voir encadré), et des Etats de la zone euro, via l'Official Sector Involvement (OSI). Ce dernier prend la forme d'un deuxième plan d'aide à la Grèce, sous l'égide du FESF et du FMI, pour un montant total de 130 milliards d'euros, après le premier plan d'aide de mai 2010 de 110 milliards. Sur la base de ce premier plan d'aide, la Grèce a pour le moment reçu 73 milliards d'euros, versés en six tranches. Dans le second plan, le FESF et le FMI se sont engagés non seulement à verser les fonds du premier plan non encore débloqués, mais également 130 milliards d'euros pour la période 2012-2014. Contrairement au premier programme d'aide, le financement sera assuré par le FESF, plutôt que par des prêts bilatéraux.

Encadré : Opération réussie pour le plan PSI (Private Sector Involvement)

Décidé au sommet du 26 octobre 2011, le plan PSI a été lancé le 24 février 2012. Sur une dette souveraine de 368 milliards d'euros, le montant de la dette grecque concerné par cet échange atteint 206 milliards d'euros.

\section{Que prévoit cet échange?}

En échange d'une obligation, les créanciers recevront :

- une nouvelle obligation grecque, de maturité 30 ans, dont le montant nominal représentera $31,5 \%$ de l'obligation initiale. Ces nouvelles obligations auront pour échéance 2042, avec un coupon step up, évoluant par paliers au cours du temps: le coupon annuel sera de $2 \%$ jusqu'en 2015, puis $3 \%$ en 2020, 3,65 \% jusqu'en 2021, et 4,3\% ensuite ;

- des titres du FESF avec une maturité de un ou deux ans, et ayant une valeur nominale de $15 \%$ de l'obligation initiale ;

- concernant les coupons courus sur les obligations échangées, ils seront payés par le FESF par l'émission d'obligations de court terme ; 
- enfin, les participants à l'opération recevront des titres indexés sur le PIB grec. Ces titres donneront lieu au versement d'un coupon pouvant atteindre jusqu'à $1 \%$ à partir de 2015 si le PIB a dépassé un certain niveau.

Les nouvelles obligations sont régies par le droit britannique. En termes nominaux, la perte essuyée à court terme par les investisseurs est de 53,5\%, mais en termes de valeur nette actuelle, la décote sera en réalité comprise entre $70 \%$ et $75 \%$, ce qui en fait une restructuration comparable à celle de l'Argentine, lors de l'échange de dette de 2005 .

\section{Le succès de l'échange}

Sur un montant total de 206 milliards d'euros soumis à l'échange, les obligations soumises au droit grec représentaient 177 milliards d'euros (86 \% du total), les obligations de droit international représentant 29 milliards. Au total, le 9 mars 2012, 83,5\%11 des créanciers privés $(85,8 \%$ des créanciers pour les obligations de droit grec, mais seulement $69 \%$ pour les obligations de droit étranger) ont accepté l'échange d'obligations souveraines pour un montant de 172 milliards d'euros. Concernant les titres de droit grec en circulation, les clauses d'action collective, permettant à la Grèce d'obliger les investisseurs à accepter l'échange, pourraient s'appliquer et conduire l'Etat à récolter 26 milliards d'euros supplémentaires (participation des créanciers privés de $100 \%$ sur les titres de droit grec). En outre, la date de clôture pour l'échange des obligations de droit international ayant été repoussée au 24 mars 2012, la participation pourrait encore augmenter.

En novembre 2011, le nouveau gouvernement de Lucas Papademos a pris les commandes de la Grèce. Nous faisons l'hypothèse que le gouvernement tiendra ses objectifs de réduction du déficit primaire pour 2012 et 2013, même si cela doit nécessiter de nouveaux plans d'ajustement en cours d'année. Les objectifs de déficit pour 2012 et pour les années suivantes ont été révisés depuis notre précédente prévision. L'objectif est d'atteindre un solde primaire de $-1 \%$ du PIB en 2012 (contre $-2,4 \%$ en 2011) et un excédent primaire de 1,8 \% en 2013. En outre, grâce au deuxième plan d'aide FESF/FMI, et au plan PSI avec les créanciers privés, la Grèce devrait voir le taux d'intérêt moyen baisser d'un point à $6,3 \%$ en 2012. Le véritable problème reste la récession qui se prolonge en 2012 et qui nécessite d'importantes restrictions supplémentaires pour une réduction limitée du déficit budgétaire.

Pour l'année 2012, le total des mesures votées se traduit par une impulsion budgétaire qui atteindrait 4,3 points de PIB, avec 9 milliards d'économies supplémentaires par rapport à 2011. Ainsi, non seulement les mesures prévues dans la Medium Term Fiscal Strategy ${ }^{12}$ (MTFS, voir prévision d'octobre 2011) auraient un impact en 2012, mais en outre, les nouvelles mesures représenteraient 2,9 point de PIB, avec la réduction du seuil d'imposition pour l'impôt sur le revenu et la fin de certaines exemptions (2,2 points), la baisse des salaires dans la fonction publique d'État, et la baisse des pensions. En outre, en février 2012, le gouvernement grec a adopté un nouveau plan de rigueur, pour un total de 1,5 point de PIB, portant exclusivement sur la réduction des dépenses, pour garantir l'objectif de déficit primaire en 2012. Parmi ces mesures, citons la réduction des dépenses de santé ( 0,5 point de PIB), la réduction des salaires dans les régimes spéciaux de $12 \%$ en moyenne, l'application de la règle de remplacement d'un fonctionnaire sur 10, une nouvelle baisse des pensions de retraite (pour les pensions supérieures à 1300 euros mensuels, baisse de 12 à $20 \%$ appliquée sur le montant excédant 1300 euros), une baisse des dépenses militaires. 
En 2013, l'atteinte de l'objectif fixé par le gouvernement nécessitera des mesures qui devront porter sur 6,4 milliards d'euros (3,2 points de PIB d'impulsion), dont seulement 1,6 milliards ont été adoptés. Un ou plusieurs nouveaux plans d'économies seront donc nécessaires pour satisfaire l'objectif de réduction du déficit. Dans cette perspective, la Grèce a prévu de mettre à jour la MTFS en mai 2012, et le gouvernement a lancé une révision générale des dépenses publiques : on s'attend à une réduction des dépenses de transfert et du budget de la défense, à la restructuration des administrations centrale et locale et à la poursuite des licenciements dans la fonction publique.

Les privatisations devraient également s'accélérer en 2012 et 2013 : elles rapporteraient 9 milliards d'euros cumulés en 2013, contre 1,5 milliards d'euros début 2012, l'objectif d'arriver à 50 milliards en 2015 ayant finalement été abandonné.

\section{Portugal : condamné à éviter le sort grec}

Étant donné le contexte récessif (recul du PIB de 1,5\% en 2011), les mesures de restriction se sont avérées insuffisantes pour respecter l'objectif de déficit annoncé pour 2011 (à savoir 5,9\% du PIB, après 9,8\% en 2010) et les autorités portugaises ont dû avoir recours à des mesures exceptionnelles. Le déficit pour 2011 n'est pas encore définitif, mais le ministre des Finances a annoncé qu'il serait de l'ordre de $4 \%$ du PIB, grâce au transfert des fonds de pension des banques vers la Sécurité sociale ${ }^{13}$. Sans cette mesure, le déficit aurait été de 7,5\% du PIB.

En 2012, l'impulsion budgétaire serait à nouveau très négative (-3 points selon nos prévisions), dont les deux tiers basés sur des réductions de dépenses. Parmi ces dernières, les $13^{\mathrm{e}}$ et $14^{\mathrm{e}}$ mois des fonctionnaires et retraités dont les revenus sont supérieurs à 1100 euros par mois vont être supprimés, la réduction de l'emploi et de l'investissement publics va se poursuivre, des économies sont prévues à différents niveaux de l'administration et l'accès aux prestations sociales sera durci. Les recettes devraient bénéficier de la hausse de la TVA dans différents secteurs, comme la restauration (via un passage du taux intermédiaire de $13 \%$ au taux normal de $23 \%$ ), des hausses des taxes sur l'énergie, le tabac, l'alcool et les voitures, de l'augmentation de l'imposition des revenus du capital et de la réduction de diverses exemptions fiscales pour les ménages et les entreprises. En 2013, nous avons fait l'hypothèse d'une impulsion budgétaire de -3 points. Comme en 2012, I'austérité s'appuiera sur une baisse de l'emploi public et de l'investissement, des coupes de dépenses dans la santé et un élargissement de la base fiscale via l'élimination de déductions.

La récession devrait s'amplifier en 2012 (-2,9\%) et la croissance être faible en 2013 (0,2\%). Les objectifs de déficit public (4,5\% du PIB en 2012, $3 \%$ en 2013) seraient néanmoins presque respectés selon nos prévisions, grâce aux recettes des privatisations. Nous prévoyons ainsi un déficit de 4,5\% du PIB en 2012 puis 3,5\% en 2013. Sur les 5 milliards environ de recettes attendues des privatisations, un peu plus de 3 milliards (1,8 point de PIB) sont d'ores et déjà actés avec principalement la vente d'entreprises du secteur énergétique. Les opérations ayant été réalisées fin 2011, les ressources concerneront essentiellement l'année 2012. Selon les prévisions officielles,

13. En échange, le gouvernement devra à l'avenir prendre en charge les pensions des bénéficiaires de ces fonds de pension. 
en 2011, 0,4 point de PIB de recettes de privatisations seront comptabilisées et 2,3 points en 2012 (en tenant compte des prochaines privatisations). En 2013, les privatisations ne représenteraient plus que 0,6 point de PIB.

Malgré les efforts budgétaires réalisés par le Portugal, des doutes subsistent sur sa capacité à pouvoir réemprunter à long terme à partir de fin 2013, les taux obligataires à 10 ans restant à des niveaux élevés $(13,6 \%$ sur les quinze premiers jours de mars 2012). De ce fait, une nouvelle aide serait nécessaire. Depuis mai 2011, I'État portugais n'emprunte plus sur les marchés financiers que sur des maturités courtes ( 3 et 6 mois et plus récemment un an); les maturités longues étant assurées par les prêts européens et du FMI, au taux de 3,5\%. Début 2012, le Portugal avait reçu 36,5 milliards de prêts sur les 78 prévus par la Commission européenne, le FESF et le FMI en mai 2011. La prochaine tranche d'environ 15 milliards devrait être versée en avril 2012, la mission conjointe de la Commission européenne, de la BCE et du FMI fin février ayant été satisfaite des mesures prises en 2011 (réformes du marché du travail, mesures de consolidation budgétaire...).

\section{La BCE évite le pire}

L'évolution de l'écart critique, mesurant la différence entre le taux d'intérêt et la croissance nominale, joue un rôle essentiel pour la dynamique de la dette et conditionne l'ampleur des mesures d'ajustement nécessaires à la réduction des déficits budgétaires. Si les banques centrales ne déterminent pas directement le niveau des taux d'intérêt de long terme, elles peuvent, par des achats ciblés de titres, faire pression à la baisse. C'est ce que fait la BCE depuis le mois de mai 2010 en achetant directement des titres obligataires émis par les pays touchés par la crise des dettes souveraines. Ce faisant, la BCE prend soin de préciser qu'elle ne souhaite pas contribuer au financement des États mais que ses opérations sont destinées à rendre plus efficace la transmission de la politique monétaire. C'est pourquoi sa stratégie reste principalement focalisée sur les opérations de refinancement accordées au système bancaire ce qui peut également indirectement contribuer à la détente des taux d'intérêt.

Force est cependant de constater que le niveau moyen des taux d'intérêt dans la zone euro est resté plus élevé que celui des taux américain ou britannique alors même que le déficit budgétaire de la zone euro, dans son ensemble, est plus faible qu'aux États-Unis ou qu'au Royaume-Uni (graphique 6). Ce déficit devrait être de 3,6\% en 2011 dans la zone euro contre respectivement 9,7 et 8,3\% aux États-Unis et au Royaume-Uni14. Pour autant, la politique menée par la BCE n'a sans doute pas été moins efficace que celle de la Réserve fédérale et de la Banque d'Angleterre, mais surtout moins ambitieuse.

En effet, si les taux d'intérêt publics sont restés plus élevés dans la zone, c'est avant tout parce que les achats de titres publics réalisés par la BCE ont été bien moindres que les ceux de la Réserve fédérale, de la Banque d'Angleterre ou encore de la Banque du Japon (graphique 7). La BCE avait acheté, fin 2011, 211 milliards d'euros de titres de dette émis par les gouvernements grecs, portugais, espagnols et italiens, ce qui représente $2,3 \%$ du PIB de la zone euro. Aux États-Unis et au Royaume-Uni, ces portefeuilles atteignaient respectivement 1660 milliards de dollars et 200 milliards 15

14. En termes de ratio de dette publique, celle du Royaume-Uni s'élèverait à $90 \%$ en 2011 contre 95,6\% dans la zone euro et $97,6 \%$ aux États-Unis. 
de livres, soit 11 et $13 \%$ de leur PIB respectif. En dépit de cette insuffisance, la BCE aura sans doute permis de garantir la viabilité de la zone euro.

Graphique 6. Taux d'intérêt longs publics

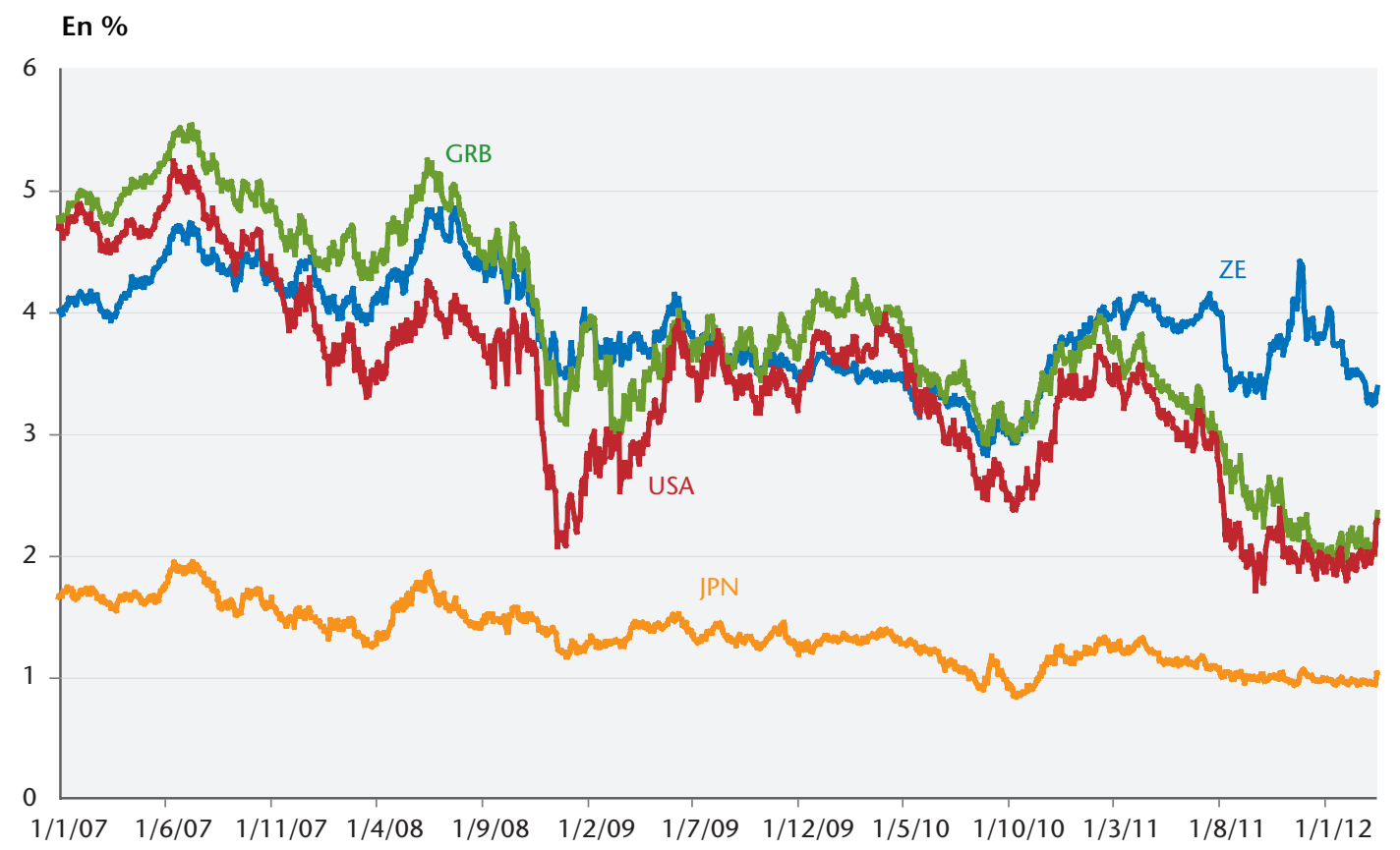

Source : Datastream.

Graphique 7. Portefeuille de titres publics des banques centrales

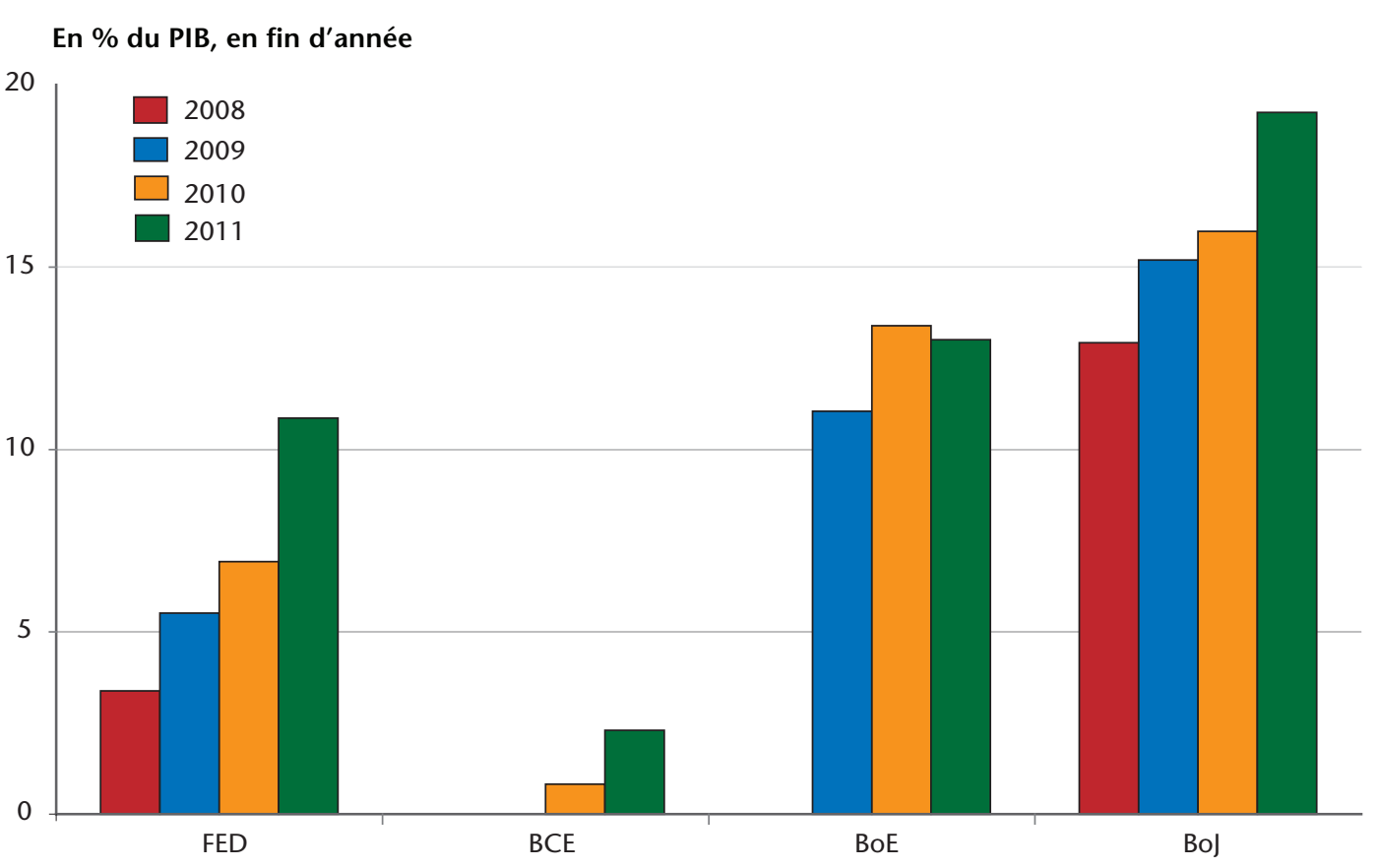

Sources: Banques centrales nationales, Comptes nationaux.

15. La Banque d'Angleterre a même décidé fin 2011 d'augmenter progressivement son portefeuille pour le porter à 250 milliards de livres. 
En complément de son action sur les marchés obligataires, la BCE a continué à soutenir le système bancaire en allouant, en deux opérations ${ }^{16}$, plus de 1000 milliards d'euros de refinancement au système bancaire pour une maturité exceptionnelle de 3 ans. Le refinancement des banques européennes sur les marchés était effectivement devenu critique au deuxième semestre 2011. L'accès au marché obligataire était de plus en plus restreint et le coût de la liquidité en blanc' ${ }^{17}$ à court terme retrouvait un niveau proche de celui précédant l'épisode de la faillite de Lehman Brother (graphique 8). Les banques - notamment les banques italiennes et espagnoles - ont donc massivement souscrit à l'offre de refinancement de la BCE, réussissant à couvrir près de $80 \%$ de leur dette arrivant à échéance entre 2012 et 201418 .

Graphique 8. Coût de la liquidité à court terme dans la zone euro

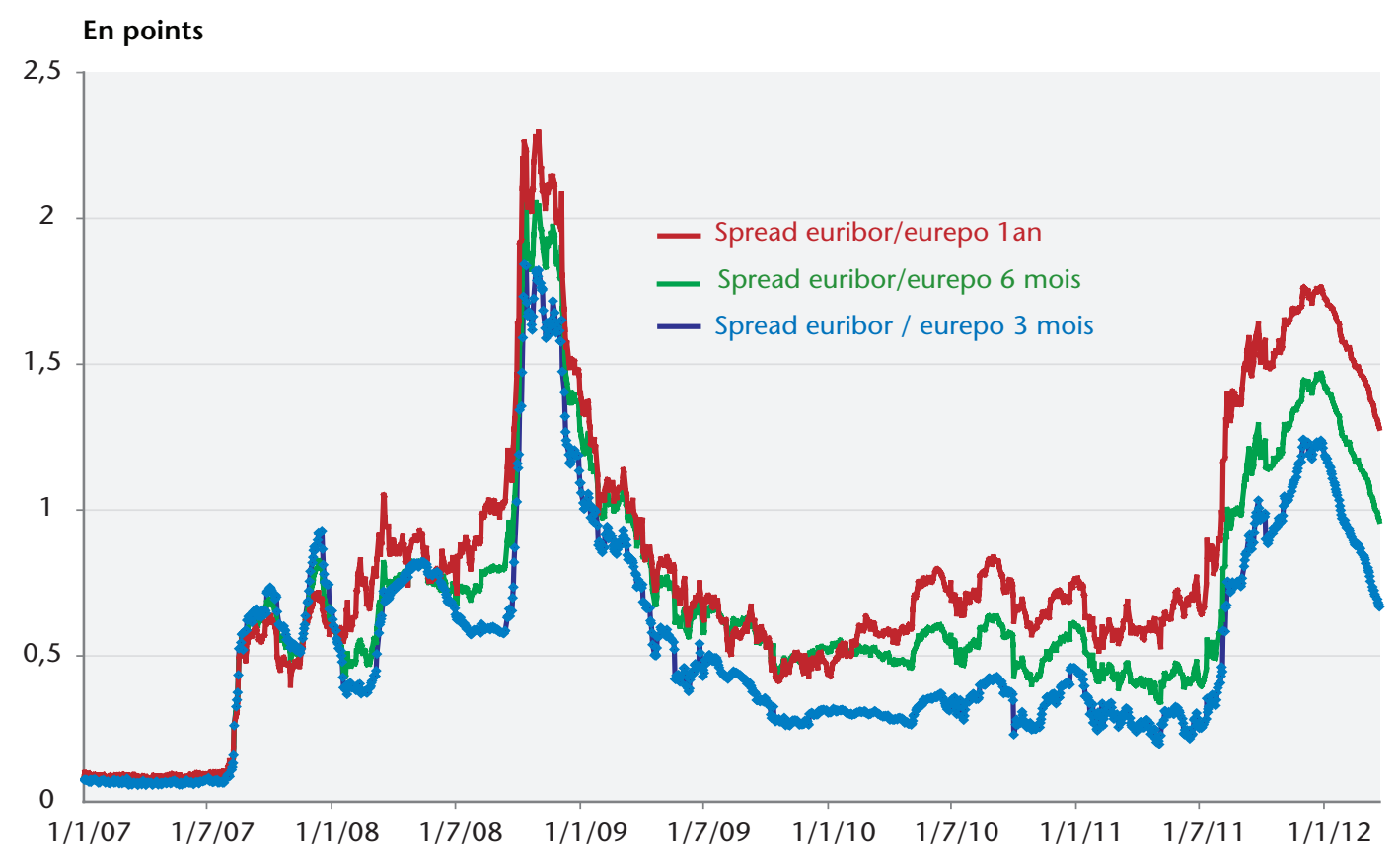

Note de lecture : les taux Euribor correspondent à des prêts interbancaires non garantis. Les taux Eurepo sont appliqués à des prêts interbancaires garantis.

Source : Datastream.

En réduisant le risque de faillite, la $B C E$ espérait restaurer la confiance dans le système bancaire, permettre aux banques de distribuer des crédits aux agents non financiers et réduire la pression sur les taux d'intérêt de long terme. Si un effet multiplicateur direct sur le crédit est loin d'être garanti, l'impact de ces mesures sur l'activité pourrait passer par un autre canal. En effet, en bénéficiant d'un coût de refinancement à $1 \%$, les banques pourront d'une part améliorer leurs marges et réduire le risque de taux lié au refinancement de leur passif. D'autre part, ces opérations contribueraient à diminuer le risque d'illiquidité. La perception globale du risque au sein du système bancaire serait alors réduite, ce qui agirait en retour sur les conditions de crédit ${ }^{19}$. Il est

16. Ces allocations ont été réalisées le 22 décembre 2011 pour un montant de 489,2 milliards d'euros et au 1er mars 2012 pour un montant de 529,5 milliards d'euros.

17. La liquidité en blanc correspond aux prêts interbancaires de court terme non garantis.

18. Voir BIS (2011) : «European bank funding and deleveraging », BIS Quaterly Review, mars, p.1-12.

19. Voir Borio \& Zhu (2008): "Capital regulation, risk-taking and monetary policy: a missing link in the transmission mechanism? », BIS Working Paper, $\mathrm{n}^{\circ} 268$. 
cependant encore prématuré pour juger de l'efficacité de ces mesures. L'enquête sur les conditions de crédit indique un nouveau durcissement depuis la fin de l'année 2011, qui serait notamment lié à des facteurs d'offre, les banques confirmant leurs difficultés de financement. Dans ces conditions, on peut craindre un nouvel épisode de resserrement du crédit. II serait néanmoins atténué par les actions de la BCE. La baisse de l'écart entre les taux Euribor et Eurepo montre en tout cas que les tensions sur le marché interbancaire ont été réduites, ce qui devrait contribuer le trimestre prochain à lever, au moins partiellement, les contraintes pesant sur le financement des banques. L'action de la BCE pourrait également avoir un effet favorable sur les taux longs publics via deux canaux. En réduisant le risque de faillite au sein du système bancaire, la probabilité que les gouvernements soient contraints d'intervenir pour renflouer une banque baisserait. Les marchés anticiperaient une amélioration de la soutenabilité de leur dette, ce qui ferait baisser la prime de risque. Par ailleurs, les banques pourraient replacer une partie de ces liquidités sur les marchés obligataires. La hausse de la demande en résultant contribuerait également à la baisse des taux d'intérêt public à long terme. L'évolution récente du portefeuille des institutions monétaires et financières de la zone euro et des taux d'intérêt confirment cette hypothèse. Les banques, notamment les banques italiennes et espagnoles 20 , ont effectué des achats de titres obligataires si bien que sur l'ensemble de la zone euro, le poids, dans leur bilan, du portefeuille de titres publics est passé de 7,2\% en septembre 2011 à 7,6\% janvier 2012 (graphique 9), ce qui correspond à un achat net de titres supérieur à 100 milliards d'euros.

Graphique 9. Crédits et titres publics des IFM de la zone euro

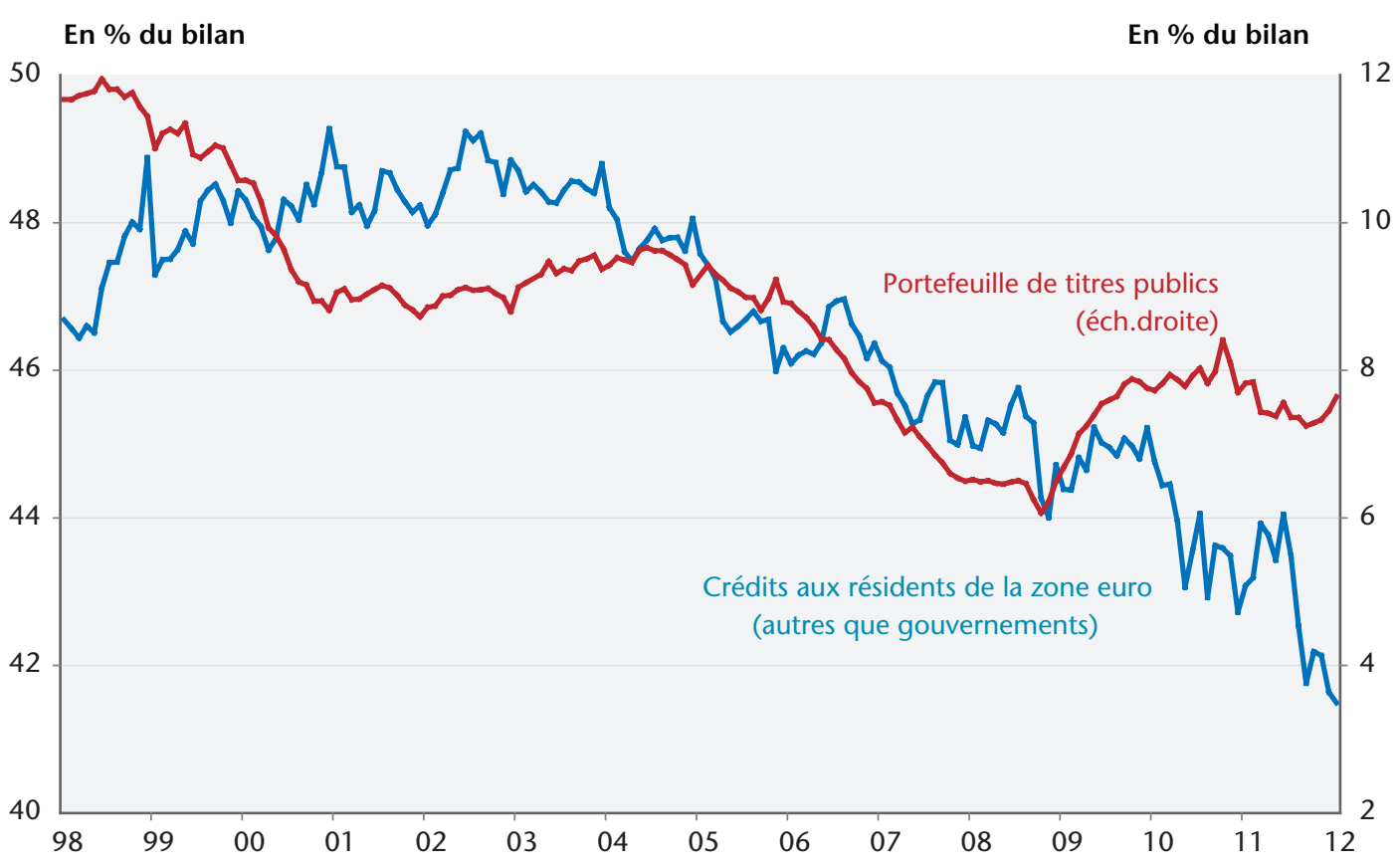

Source : $B C E$. 
II est donc probable que l'action de la BCE en décembre 2011 et mars 2012 a contribué à la détente des taux d'intérêt observée depuis le début de l'année sur les marchés obligataires. S'il s'agit d'une bonne nouvelle en matière de soutenabilité de la dette, le deuxième enjeu principal de la politique monétaire dépendra de son effet sur les conditions de crédits. Les taux d'intérêt appliqués aux crédits sont effectivement repartis à la hausse depuis avril 2010, en lien notamment avec la crise des dettes souveraines. Le maintien d'une politique monétaire accommodante reste indispensable dans la zone euro. En se concentrant sur la fragilité bancaire, la BCE tient compte de l'importance de ce secteur dans le financement de l'économie. Elle prend les mesures qui devraient atténuer les contraintes pesant sur l'offre de crédit mais il reste que le biais trop restrictif de la politique budgétaire réduit les perspectives de croissance, ce qui contraint de fait la demande de crédit.

\section{Entreprises : de la dégradation des marges aux gains de compétitivité}

La Grande Récession de 2008/2009 a creusé l'écart entre la production potentielle et la production réalisée, écart qui ne s'est pas refermé depuis. La reprise entamée au cours de l'année 2010 a fait long feu et les perspectives de croissance ont été revues à la baisse. Face à cette atonie de la demande, les entreprises sont contraintes de poursuivre l'ajustement de leurs coûts de production, en particulier les coûts salariaux. L'ajustement peut se faire par la quantité (destructions d'emplois) ou par les prix (modération salariale). La contraction de la main-d'œuvre aide les entreprises à reconstituer leurs marges, tandis que la maîtrise des coûts salariaux leur permet en outre de gagner en compétitivité à l'exportation, ce qui dans un contexte d'atonie de la demande intérieure pourrait s'avérer être le seul moteur de croissance. Nous analysons donc l'évolution de la productivité, des taux de marge et de la compétitivité-prix des entreprises de la zone euro dans le secteur marchand depuis le début de la crise.

\section{L'ajustement de la productivité se poursuit}

Durant la première phase de la crise, soit du premier trimestre 2008 au premier trimestre 2010, la valeur ajoutée du secteur marchand s'est effondrée brutalement dans tous les pays de la zone euro, mais certains pays - Allemagne, Italie et Espagne ont été plus touchés que la France (tableau 8). Si les destructions d'emploi ont été massives durant cette période, elles ont été moins fortes que la chute de la production. La productivité s'est donc dégradée dans tous les grands pays sauf en Espagne. Au cours de ces deux années de crise, l'Espagne a détruit 2,2 millions d'emplois, soit plus que la France, I'Allemagne et I'Italie réunies. Cette chute de I'emploi a permis à la productivité espagnole de faire un bond de $7,5 \%$ sur la période, alors qu'elle diminuait de $0,8 \%$ en France, de 5,6 \% en Allemagne et de 4,4 \% en Italie.

La deuxième phase, du premier trimestre 2010 au quatrième trimestre 2011, est celle d'un rebond de la croissance, freinée cependant par les ajustements budgétaires au sein de la zone euro et les inquiétudes entourant la crise de la dette. La valeur ajoutée marchande a rebondi en France et en Allemagne, mais reste légèrement inférieure à son niveau d'avant-crise. En Italie et en Espagne, la reprise a été plus timorée et la valeur ajoutée marchande a perdu $6 \%$ depuis le début de la crise. La croissance a 
permis de créer des emplois marchands en Allemagne (plus de 880 000) et en France (264 000), mais l'Espagne et l'Italie ont continué à détruire des emplois.

Dans les quatre pays, la productivité s'est améliorée au cours de la deuxième phase de la crise, mais le cycle de productivité reste très dégradé quelle que soit la tendance retenue (sauf en Espagne). L'Allemagne est le seul pays affichant des créations nettes d'emploi depuis début 2008, et il est aussi celui où la productivité est la plus dégradée. L'Espagne détient le triste record de destruction d'emplois marchands (plus de 3 millions), et en corollaire sa productivité a bondi de $14 \%$ depuis début 2008. La question est maintenant de savoir comment cette chute de la productivité se répercute sur les marges des entreprises.

Tableau 8. Emploi, valeur ajoutée et productivité dans le secteur marchand

\begin{tabular}{|c|c|c|c|c|c|}
\hline & & FRA & DEU & ITA & ESP \\
\hline \multicolumn{6}{|l|}{ Emploi marchand } \\
\hline & 2008 T1-2010 T1 & -523 & -321 & -695 & -2242 \\
\hline \multirow[t]{3}{*}{ En milliers de personnes } & 2010 T1-2011 T4 & 264 & 882 & -88 & -784 \\
\hline & $2008 \mathrm{~T} 1-2011 \mathrm{~T} 4$ & -259 & 561 & -783 & -3025 \\
\hline & 2008 T1-2010 T1 & $-2,7$ & $-1,1$ & $-3,1$ & $-13,0$ \\
\hline \multirow[t]{2}{*}{ En $\%$} & 2010 T1-2011 T4 & 1,4 & 3,2 & $-0,4$ & $-5,2$ \\
\hline & 2008 T1-2011 T4 & $-1,3$ & 2,0 & $-3,5$ & $-17,5$ \\
\hline \multicolumn{6}{|c|}{ Valeur ajoutée marchande (réelle) } \\
\hline & 2008 T1-2010 T1 & $-3,5$ & $-6,7$ & $-7,3$ & $-6,4$ \\
\hline & 2010 T1-2011 T4 & 3,2 & 6,5 & 1,4 & 0,4 \\
\hline & 2008 T1-2011 T4 & $-0,4$ & $-0,6$ & $-6,1$ & $-6,0$ \\
\hline \multicolumn{6}{|l|}{ Productivité marchande } \\
\hline & 2008 T1-2010 T1 & $-0,8$ & $-5,6$ & $-4,4$ & 7,5 \\
\hline & 2010 T1-2011 T4 & 1,8 & 3,2 & 1,8 & 5,9 \\
\hline & 2008 T1-2011 T4 & 0,9 & $-2,6$ & $-2,5$ & 13,9 \\
\hline
\end{tabular}

Sources : Données nationales, calculs OFCE.

\section{La modération salariale ne suffit pas à rétablir le taux de marge}

La productivité est l'un des déterminants du taux de marge des entreprises, mais I'ajustement peut aussi se faire par la réduction des coûts salariaux. Dans le tableau 9, nous avons décomposé les coûts salariaux en salaire horaire et temps de travail. Si I'on compare à la tendance des années 2000 à 2007, tous les pays sauf l'Allemagne ont enregistré un net ralentissement du salaire horaire dans le secteur marchand. L'Allemagne est un cas particulier car la modération salariale avait démarré dès le début de la décennie, avec une hausse du salaire horaire nominal de 1,6 \% par an en moyenne, contre 3,4\% en France, 3,5\% en Italie et 4,5\% en Espagne.

La progression des salaires réels est restée contenue en dessous de 0,7\% annuellement durant la première phase dans tous les pays sauf la France. Depuis début 2010, la France se démarque encore de ses partenaires car elle est la seule à ne pas enregistrer 
de baisse du salaire horaire réel. Cette baisse est particulièrement violente (près de $5 \%$ en 6 trimestres) en Italie et en Espagne. La réduction du nombre moyen d'heures travaillées par salarié a permis de limiter la progression des coûts salariaux dans tous les pays sauf en Espagne, où le nombre moyen d'heures travaillées a augmenté du fait de la suppression d'un grand nombre d'emplois à temps partiel. La réduction du temps de travail a surtout eu lieu durant la première phase de la crise. En Allemagne, la reprise de l'emploi s'est accompagnée d'une hausse du nombre d'heures travaillées, dégradant davantage le taux de marge des entreprises.

Le taux de marge allemand apparaît comme le plus dégradé depuis le début de la crise $(-4,8 \%)$, suivi du taux de marge français $(-4,1 \%)$. En France, la baisse du taux de marge est principalement imputable au dynamisme des salaires malgré la crise, tandis qu'en Allemagne, c'est la chute de la productivité en 2008 qui en est responsable. Mais si l'on observe l'évolution des taux de marge sur une période plus longue (graphique 10), c'est l'Italie qui se retrouve en plus mauvaise posture. La productivité tendancielle y est plus faible qu'en France, mais les salaires progressaient aussi vite avant la crise. Depuis 2008, la modération salariale ainsi que la baisse des heures travaillées ont permis de limiter la dégradation du taux de marge, mais celui-ci était déjà bas avant la crise. L'Espagne au contraire a vu son taux de marge progresser fortement depuis 2008, du fait de l'envolée de la productivité.

Tableau 9. Emploi, valeur ajoutée et productivité dans le secteur marchand

Glissement, en \%

\begin{tabular}{|c|c|c|c|c|}
\hline & FRA & DEU & ITA & ESP \\
\hline \multicolumn{5}{|l|}{ Salaire horaire nominal } \\
\hline 2008 T1-2010 T1 & 4,9 & 2,9 & 3,4 & 2,8 \\
\hline 2010 T1-2011 T3 & 3,9 & 2,3 & 1,8 & 1,3 \\
\hline 2008 T1-2011 T3 & 9,0 & 5,2 & 5,2 & 4,1 \\
\hline \multicolumn{5}{|l|}{ Salaire horaire réel } \\
\hline 2008 T1-2010 T1 & 2,7 & 1,3 & 0,7 & 1,0 \\
\hline 2010 T1-2011 T3 & 0,0 & $-1,6$ & $-4,9$ & $-4,8$ \\
\hline 2008 T1-2011 T3 & 2,8 & $-0,3$ & $-4,3$ & $-3,9$ \\
\hline \multicolumn{5}{|l|}{ Temps de travail (en heures) } \\
\hline 2008 T1-2010 T1 & $-2,0$ & $-1,9$ & $-1,5$ & 0,8 \\
\hline 2010 T1-2011 T3 & 0,1 & 1,8 & $-0,3$ & 2,4 \\
\hline 2008 T1-2011 T3 & $-1,9$ & $-0,2$ & $-1,8$ & 3,2 \\
\hline \multicolumn{5}{|l|}{ Taux de marge } \\
\hline 2008 T1-2010 T1 & $-3,3$ & $-5,0$ & $-2,4$ & 4,7 \\
\hline 2010 T1-2011 T3 & $-0,9$ & 0,2 & 0,9 & 3,2 \\
\hline 2008 T1-2011 T3 & $-4,1$ & $-4,8$ & $-1,6$ & 8,1 \\
\hline
\end{tabular}

Sources : Eurostat, OCDE, calculs OFCE.

Finalement, il semble que c'est surtout en France et en Italie que I'ajustement devra se poursuivre. Même en cas de reprise, la progression de l'emploi sera freinée jusqu'à la fermeture du cycle de productivité. Quant aux négociations salariales, elles seront difficiles tant que les entreprises n'auront pas retrouvé un niveau de marge jugé viable. Cependant, la compression des coûts salariaux pourrait permettre aux entreprises d'améliorer leur compétitivité et de gagner des parts de marché à l'exportation. L'ajus- 
tement de la productivité se poursuivrait également en Allemagne. L'impact sur le marché de l'emploi ne se poserait cependant pas de la même façon dans la mesure où I'Allemagne a quasiment retrouvé le plein emploi. Dans ces conditions, I'ajustement du cycle de productivité laisserait plutôt des marges de manœuvre pour les négociations salariales.

Graphique 10. Évolution du taux de marge dans le secteur marchand

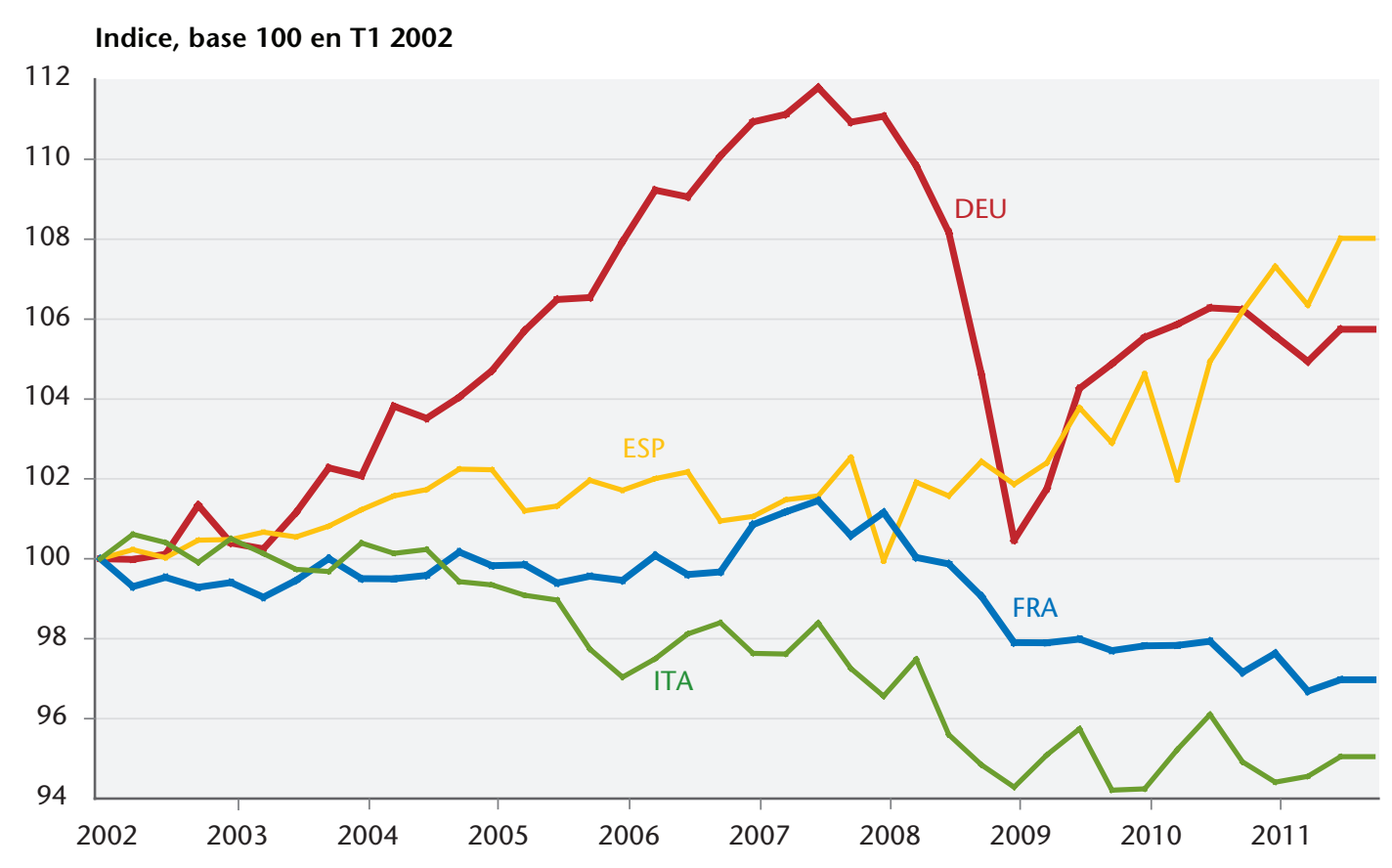

Sources : Eurostat, OCDE, calculs OFCE.

\section{Compétitivité cô̂t : des évolutions contrastées}

Comme on l'a vu précédemment, face à la crise, les entreprises ont ajusté leurs coûts autant que possible pour limiter la dégradation de leur taux de marge et rester compétitives. En s'intéressant aux coûts salariaux unitaires (CSU) dans I'industrie des différents pays de la zone euro, on constate que la crise a modifié en profondeur leurs évolutions. Pour notre analyse, nous nous appuyons sur les indicateurs de la Commission européenne qui calcule les CSU de chaque pays relativement à l'ensemble de la zone euro. Les CSU diminuent quand la productivité par tête augmente plus vite que le coût salarial par tête relativement aux autres pays de la zone euro. Entre le début de la crise et le troisième trimestre 2011, seule I'Allemagne a vu ses CSU se dégrader franchement par rapport aux autres pays (graphique 11). En Italie, en France et au Portugal, les CSU ont peu varié entre le début de la crise et fin 2011. En revanche, en Espagne, en Estonie, en Irlande et en Grèce, la tendance est à la baisse des CSU relativement à leurs partenaires de la zone euro. Pour I'Espagne, I'Estonie et I'Irlande, la rupture est nette avec les années 2002-2007 où leurs CSU s'étaient dégradés. En Grèce, la détérioration était récente après des années de stabilité relative. Ainsi dans ces quatre derniers pays, les destructions d'emplois, la modération, voire la déflation salariale $^{21}$, se sont traduites par des gains de compétitivité-coût. 
Graphique 11. Coût salarial unitaire dans l'industrie par rapport à la zone euro

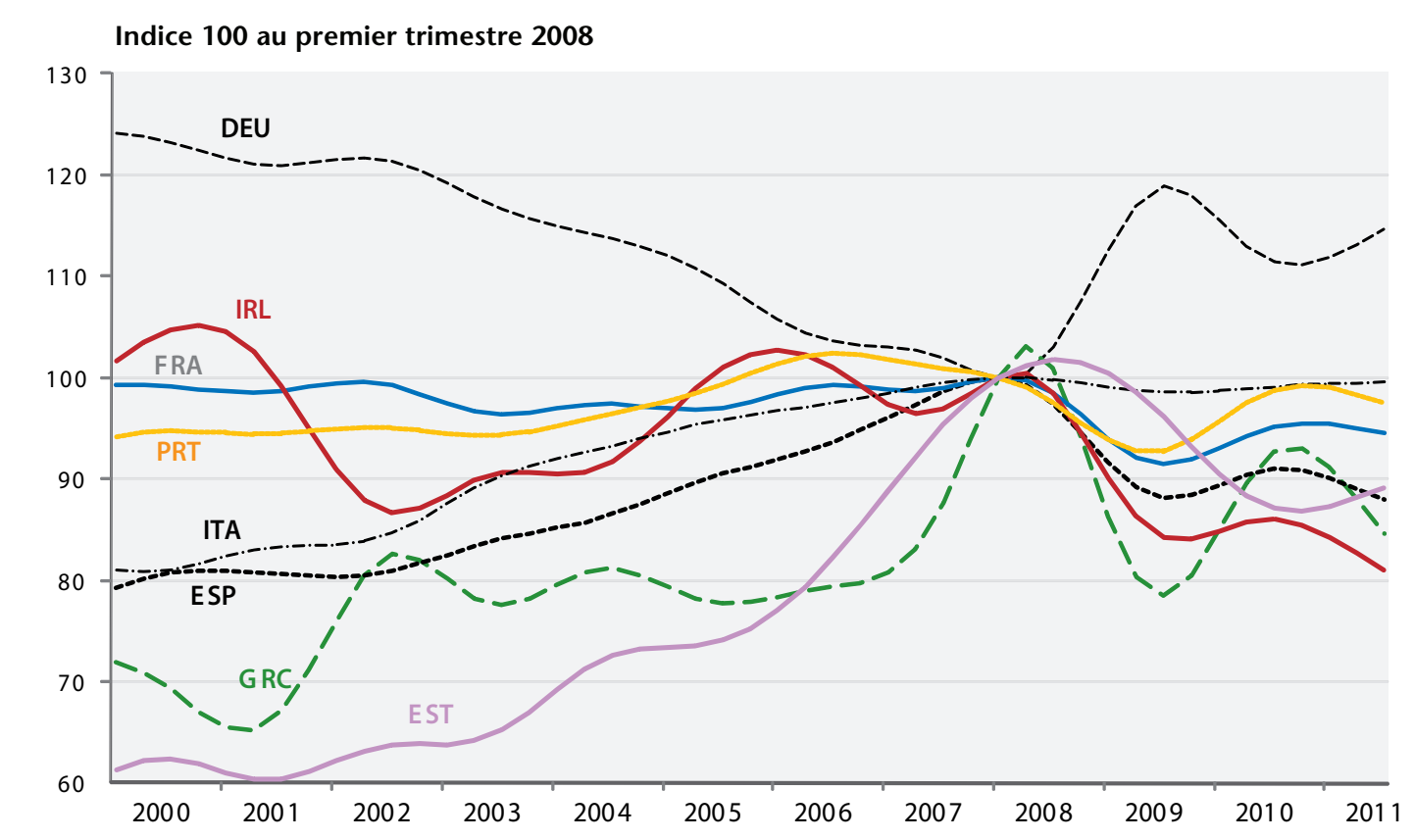

Source : Commission européenne.

L'analyse des prix relatifs des exportations de biens et services donne une vision un peu différente. Certes, le champ n'est pas exactement le même, puisque les exportations de services sont intégrées, mais cet indicateur permet de conclure sur la compétitivité-prix indépendamment des coûts des entreprises. On constate ainsi que les prix des entreprises allemandes baissent relativement aux autres, en raison d'efforts sur les marges à l'exportation (graphique 12). La compétitivité-prix des produits s'améliore aussi en France, en Espagne et en Irlande, en cohérence avec l'évolution de la compétitivité-coût. En Estonie et surtout au Portugal, les baisses de CSU ne se répercutent pas ou peu sur les prix, les entreprises choisissant de reconstituer leurs marges. Cela a aussi été le cas en Grèce jusqu'à fin 2010, avant que les prix ne s'infléchissent.

En cohérence avec les prix relatifs, les exportations de marchandises ont plus ou moins augmenté en 2010 et 2011, au moment où le commerce mondial redémarrait. L'Espagne, la Grèce et I'Irlande pourraient avoir bénéficié des baisses de prix relatifs, puisque les exportations ont fortement augmenté depuis le niveau d'avant-crise. En Allemagne et au Portugal, les exportations sont un peu supérieures à leur niveau de début 2008 ; en France elles ont presque rejoint ce niveau. Le Portugal apparaît alors comme un cas atypique dans la mesure où ses performances sont proches de celles de pays ayant réalisé des ajustements plus importants sur leurs prix à l'exportation. Seules les exportations italiennes restent à la traîne, en lien avec une compétitivité-prix relative en dégradation.

21. Si les coûts salariaux horaires ont reculé dans plusieurs pays depuis 2009, l'ajustement dans l'industrie a été faible par rapport aux autres secteurs (administrations publiques et construction essentiellement). Les coûts salariaux horaires dans l'industrie (y compris primes) ont baissé de 0,4\% au Portugal, de 0,8\% en Estonie, de 3,1\% en Irlande et de 4,9\% en Grèce. Pour une analyse détaillée du cas estonien, voir Levasseur (2011): «Labour market adjustments in Estonia during the global crisis », OFCE Working Paper, $\mathrm{n}^{\circ} 25$. 
Graphique 12. Prix des exportations de biens et services par rapport à la zone euro Indice 100 au premier trimestre 2008

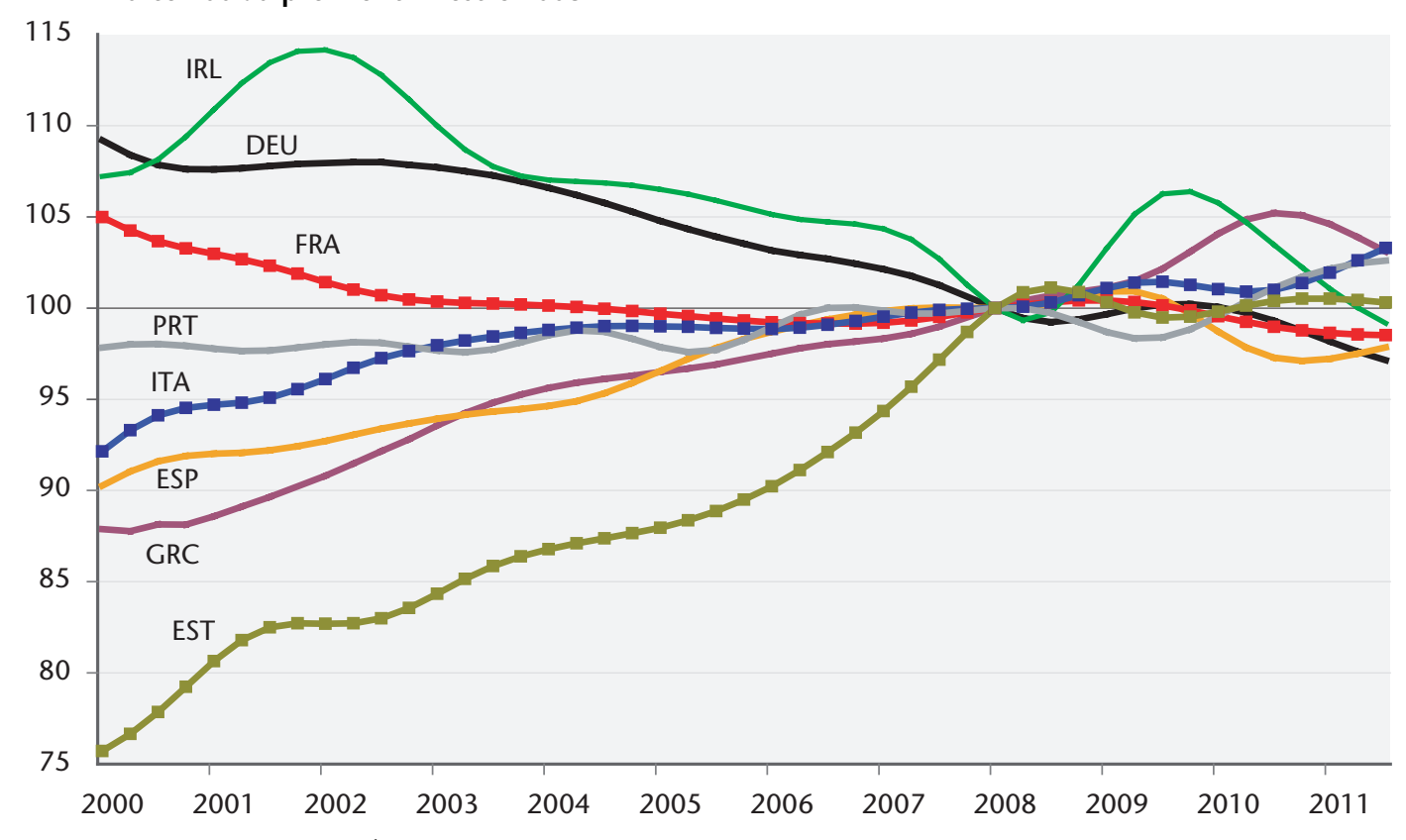

Source : Commission européenne.

Les membres d'une union monétaire ne pouvant recourir à l'arme du taux de change ne peuvent espérer regagner en compétitivité que par le biais d'un ajustement des coûts et des prix. Dans un contexte où plusieurs pays de la zone euro sont confrontés à une crise budgétaire (notamment l'Espagne, I'Irlande, la Grèce, le Portugal et I'Italie), l'austérité ne permet pas à la demande interne de jouer son rôle de moteur de la croissance. Les espoirs de reprise sont donc liés à la contribution du commerce extérieure et donc à la compétitivité. Or, si des gains de compétitivité-coût ont bien été observés dans les pays en crise, ils n'ont pas forcément été répercutés sur les prix, les entreprises préférant dans certains cas reconstituer leurs marges. II reste qu'un rebond timide des exportations atténuerait l'impact récessif des mesures d'austérité. Ces effets sont cependant insuffisants pour permettre le retour de la croissance et la décrue du chômage. D'une part, les ajustements de coûts et de prix relatifs sont lents. D'autre part, dans un contexte de synchronisation des plans d'ajustement, la demande adressée est elle-même fortement comprimée, ce qui réduit le bénéfice que les pays pourraient tirer de leurs gains de compétitivité. 
Tableau 10. Zone euro : résumé des prévisions

Variations par rapport à la période précédente, en \%

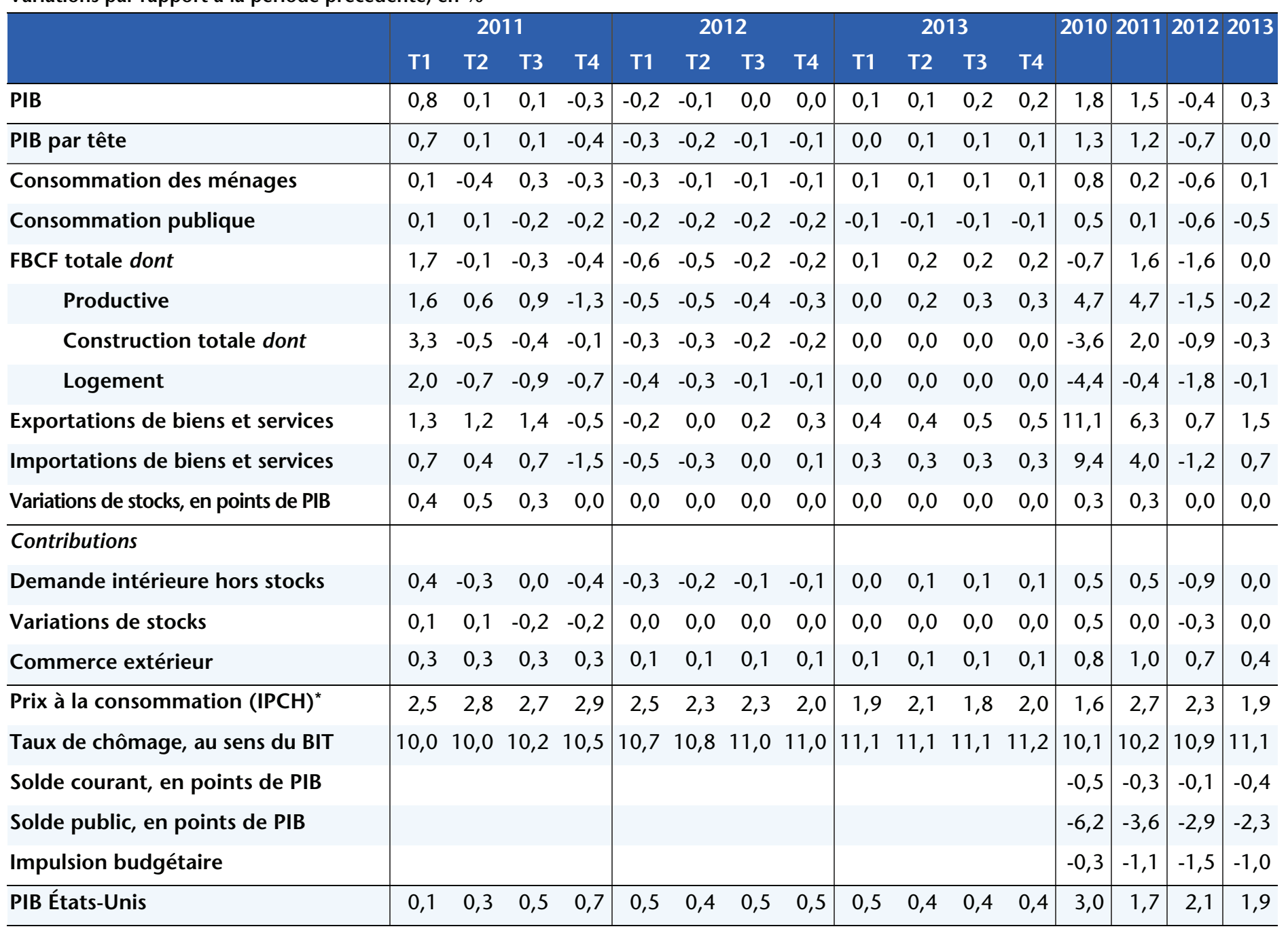

* Pour les trimestres, glissement annuel. Pour les années, moyenne annuelle.

Sources : Eurostat, calculs de l'auteur, calculs et prévisions OFCE mars 2012. 
Tableau 11. France : résumé des prévisions

Variations par rapport à la période précédente, en \%

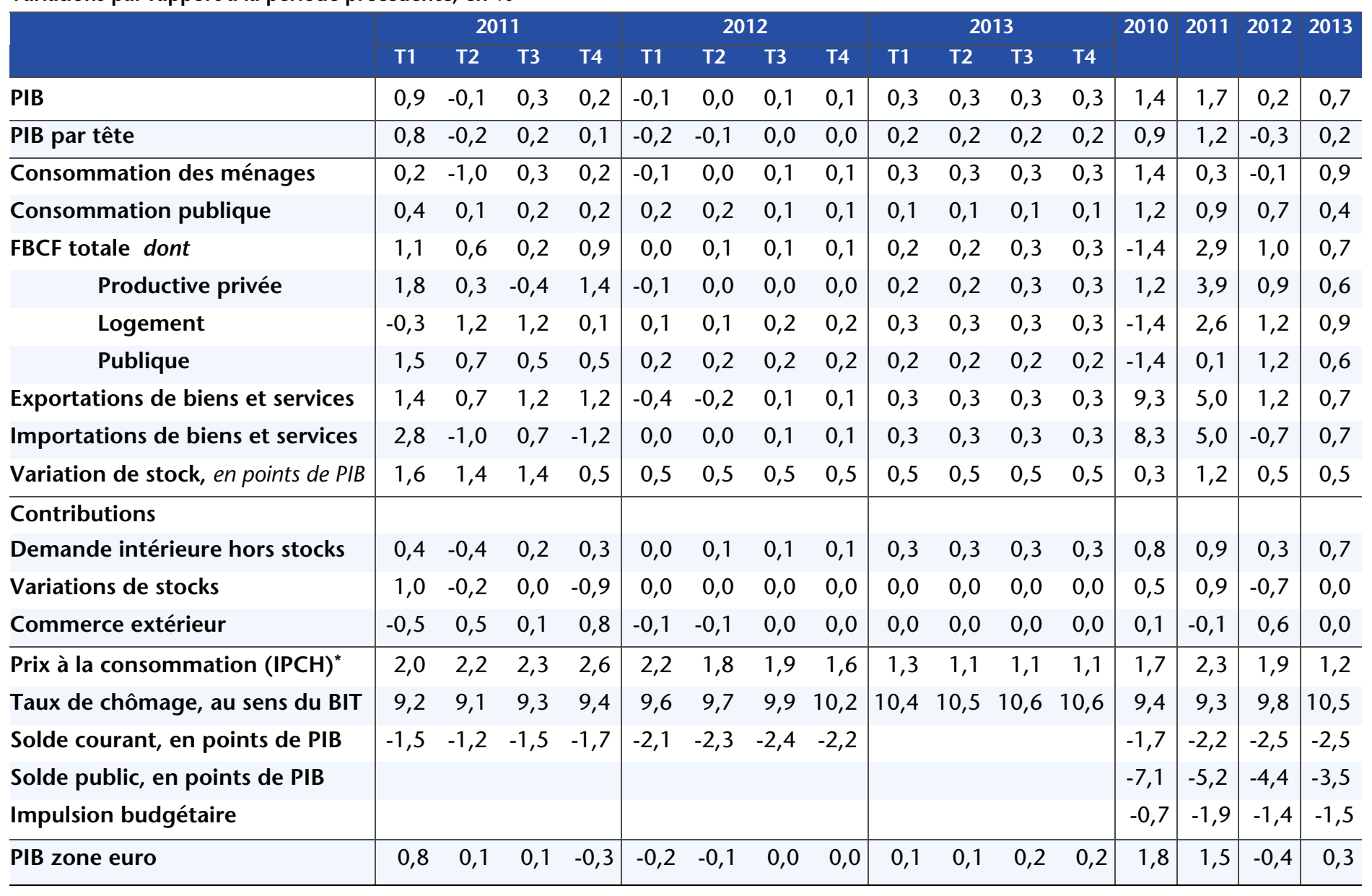

* Pour les trimestres, glissement annuel. Pour les années, moyenne annuelle.

Sources: INSEE, calculs et prévisions OFCE mars 2012. 